\title{
VORTEX2 Observations of a Low-Level Mesocyclone with Multiple Internal Rear-Flank Downdraft Momentum Surges in the 18 May 2010 Dumas, Texas, Supercell*
}

\author{
PATRICK S. SKINNER AND CHRISTOPHER C. WEISS \\ Texas Tech University, Lubbock, Texas \\ MICHAEL M. FRENCH \\ NOAA/National Severe Storms Laboratory, Norman, Oklahoma \\ HOWARD B. BLUESTEIN \\ University of Oklahoma, Norman, Oklahoma \\ PAUl M. MARKOWSKi AND YVETTE P. RichaRdson \\ The Pennsylvania State University, University Park, Pennsylvania
}

(Manuscript received 23 July 2013, in final form 28 February 2014)

\begin{abstract}
Observations collected in the second Verification of the Origins of Rotation in Tornadoes Experiment during a 15-min period of a supercell occurring on 18 May 2010 near Dumas, Texas, are presented. The primary data collection platforms include two Ka-band mobile Doppler radars, which collected a near-surface, short-baseline dual-Doppler dataset within the rear-flank outflow of the Dumas supercell; an X-band, phasedarray mobile Doppler radar, which collected volumetric single-Doppler data with high temporal resolution; and in situ thermodynamic and wind observations of a six-probe mobile mesonet.

Rapid evolution of the Dumas supercell was observed, including the development and decay of a low-level mesocyclone and four internal rear-flank downdraft (RFD) momentum surges. Intensification and upward growth of the low-level mesocyclone were observed during periods when the midlevel mesocyclone was minimally displaced from the low-level circulation, suggesting an upward-directed perturbation pressure gradient force aided in the intensification of low-level rotation. The final three internal RFD momentum surges evolved in a manner consistent with the expected behavior of a dynamically forced occlusion downdraft, developing at the periphery of the low-level mesocyclone during periods when values of low-level cyclonic azimuthal wind shear exceeded values higher aloft. Failure of the low-level mesocyclone to acquire significant vertical depth suggests that dynamic forcing above internal RFD momentum surge gust fronts was insufficient to lift the negatively buoyant air parcels comprising the RFD surges to significant heights. As a result, vertical acceleration and the stretching of vertical vorticity in surge parcels were limited, which likely contributed to tornadogenesis failure.
\end{abstract}

\section{Introduction}

A proliferation of in situ and mobile Doppler radar observations within the rear-flank downdraft (RFD) of

\footnotetext{
* Supplemental information related to this paper is available at the Journals Online website: http://dx.doi.org/10.1175/MWR-D-13-00240.s1.

Corresponding author address: Patrick Skinner, Atmospheric Science Group, Department of Geosciences, Texas Tech University, Box 41053, Lubbock, TX 79409-1053.

E-mail: patrick.skinner@ttu.edu
}

supercells following the first Verification of the Origins in Rotation in Tornadoes Experiment (VORTEX) (Rasmussen et al. 1994) has resulted in the regular identification of a small-scale region of enhanced momentum and an associated convergence boundary within the broad RFD referred to herein as an internal RFD momentum surge (or simply an RFD surge). This feature was first discussed in detail and labeled as an "RFD surge" or "second surge" by Finley and Lee (2004) and Lee et al. (2004), though it was previously briefly discussed and has subsequently been identified using many different monikers, including "outflow surge" (Dowell and 
Bluestein 2002b), "surging RFD" (Markowski et al. 2002), "jet" (Bluestein et al. 2003), "double gust front" (Adlerman 2003; Wurman et al. 2007), "embedded surge" (Hirth et al. 2008), "secondary RFD surge/gust front" (Marquis et al. 2008; Mashiko et al. 2009; Wurman et al. 2010; Skinner et al. 2011; Marquis et al. 2012; Kosiba et al. 2013; Bluestein et al. 2014), "internal RFD surge" (Finley and Lee 2008; Romine et al. 2008; Karstens et al. 2010; Lee et al. 2011; Schenkman et al. 2014), and "rear-flank downdraft internal surge" (RFDIS) (Lee et al. 2012; Karstens et al. 2013). It appears that, despite the variation in nomenclature, these terms refer to the same phenomenon.

Analysis of RFD surges has found tornadogenesis can occur just north of the surge apex (Lee et al. 2012; Kosiba et al. 2013), convergence along the RFD surge gust front may aid in tornado maintenance (Marquis et al. 2012), and tornado dissipation may coincide with an RFD surge wrapping entirely around a tornado, limiting the low-level convergence (Marquis et al. 2012) or development of a surge exhibiting large thermodynamic deficits compared to the RFD or prior RFD surges (Lee et al. 2012). Tornadoes have been observed near the gust front of an RFD surge residing entirely within the broad-scale RFD (Marquis et al. 2008, 2012), and RFD surges associated with tornadoes have exhibited small-density potential temperature deficits and large potential buoyancy compared to a representative base state (Finley and Lee 2004; Lee et al. 2004, 2011, 2012), which is similar to observations of tornadic broad-scale rear-flank downdrafts (Markowski et al. 2002; Grzych et al. 2007).

In addition to a broad RFD, which can be forced through a combination of latent chilling, hydrometeor loading, or vertical perturbation pressure gradient forces (Markowski 2002), a small, shallow, dynamically induced downdraft known as the occlusion downdraft has been identified in several numerical simulations (Klemp and Rotunno 1983; Wicker and Wilhelmson 1995; Adlerman et al. 1999) and dual-Doppler analyses (Wakimoto et al. 1998; Wakimoto and Cai 2000). The occlusion downdraft forms in response to an intensification in low-level vertical vorticity to magnitudes greater than those farther aloft, which is associated with a downward-directed vertical perturbation pressure gradient force. Each of the studies performed by Klemp and Rotunno (1983), Wicker and Wilhelmson (1995), and Adlerman et al. (1999) found that the occlusion downdraft develops within the broader-scale RFD at the surface and wraps cyclonically around the low-level circulation center with time, with at least one simulation (Adlerman 2003) producing a secondary region of enhanced convergence behind the initial RFD gust front indicative of a secondary RFD gust front bounding the occlusion downdraft. This evolution is similar to near-surface observations of internal RFD surges (Marquis et al. 2008; Wurman et al. 2010; Skinner et al. 2011; Marquis et al. 2012; Lee et al. 2012; Kosiba et al. 2013). In his review of hook echoes and rear-flank downdrafts, Markowski (2002, p. 865) described the expected near-surface manifestation of an occlusion downdraft as "a rapid, smallscale intensification of the RFD," which aptly describes the observed characteristics of internal RFD surges.

Despite the qualitative resemblance of internal RFD surges to an occlusion downdraft [as noted by Marquis et al. (2008) and Lee et al. (2012)], diagnosis of the vertical origin and forcing mechanisms of RFD surges has thus far been hampered by a paucity or complete lack of surface thermodynamic observations (Wurman et al. 2007; Marquis et al. 2008; Wurman et al. 2010; Kosiba et al. 2013), a lack of volumetric dual-Doppler observations from above the surface (Lee et al. 2011, 2012), or difficulties in accurately retrieving representative perturbation pressure fields and storm microphysics in ensemble Kalman filter (EnKF) data assimilation experiments (Marquis et al. 2012).

On 18 May 2010, a high-precipitation, tornadic supercell in the Texas Panhandle was sampled by instruments deployed by the second Verification of the Origins of Rotation in Tornadoes Experiment (VORTEX2) (Wurman et al. 2012) (Fig. 1). A nontornadic low-level mesocyclone cycle and four distinct RFD surges occurring from 2250 to 2305 UTC (all times hereafter are in UTC) were well sampled by the VORTEX2 armada, including a short-baseline dual-Doppler deployment of two Ka-band radars operated by Texas Tech University (TTUKa) (Weiss et al. 2009) and volumetric singleDoppler data collected with high temporal resolution by the Mobile Weather Radar, 2005 X-band, Phased Array (MWR-05XP) operated by the University of Oklahoma and Naval Postgraduate School (Bluestein et al. 2010). Additionally, a six-probe mobile mesonet (Straka et al. 1996) fielded by the National Severe Storms Laboratory (NSSL) and The Pennsylvania State University collected in situ observations from the near-storm environment during the radar sampling periods. The volumetric MWR-05XP observations allow for a qualitative assessment of the vertical perturbation pressure gradient force occurring coincident with RFD surge development to be made, particularly in the context of the finescale, near-surface depiction of the wind field provided by the TTUKa radars. Additionally, the failure of the observed low-level mesocyclone to produce a tornado and subsequent dissipation will be examined with regard to the thermodynamic properties of the RFD and RFD surges [e.g., Markowski et al. (2002); Grzych et al. (2007); Markowski et al. (2012a); Markowski and Richardson (2014)] and horizontal displacement 


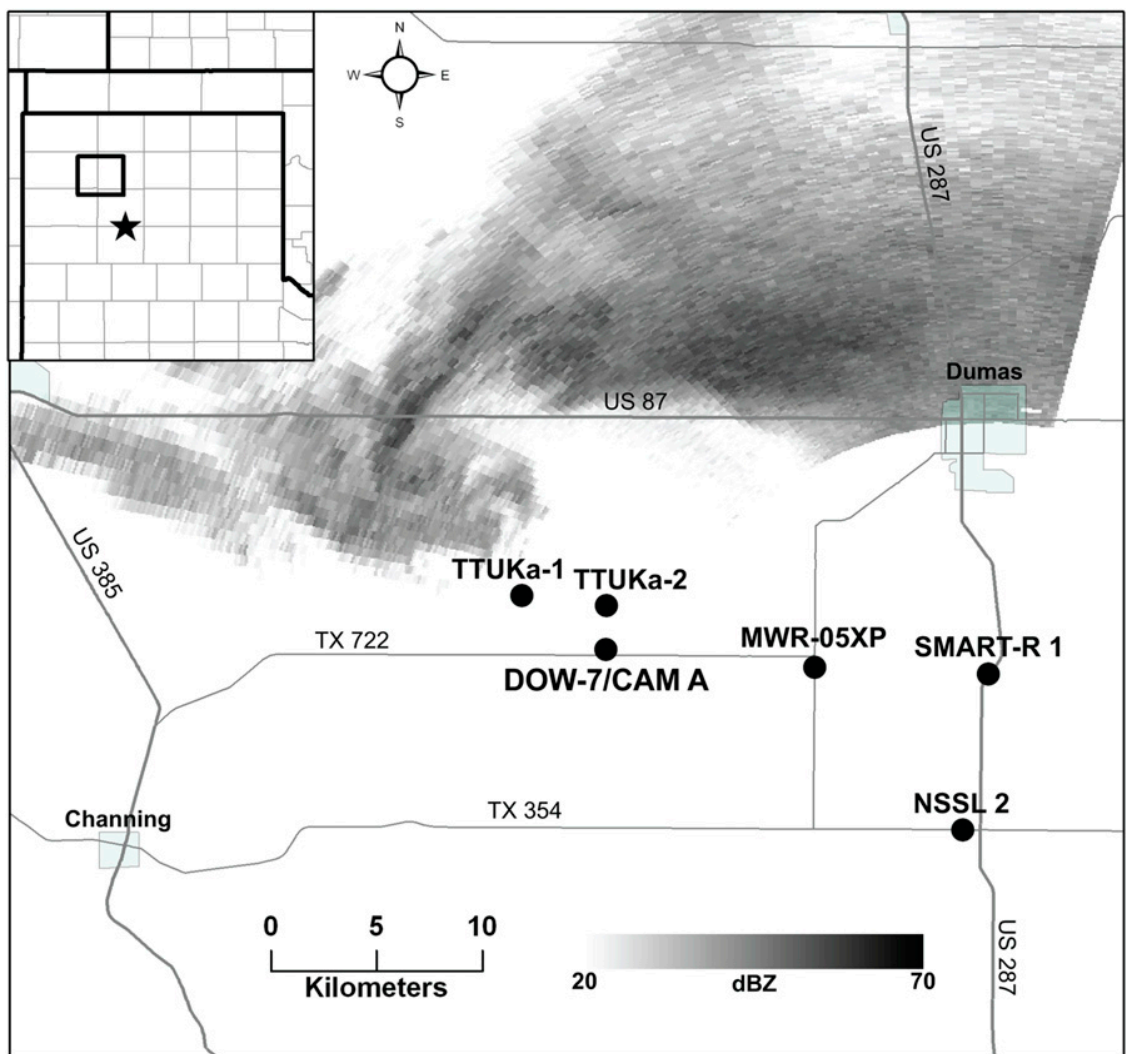

FIG. 1. Overview of VORTEX2 deployment locations during the period of interest. SMART-R $0.8^{\circ}$-elevation radar reflectivity values $>20 \mathrm{dBZ}$ at 2257:13 are overlain. Inset displays the position of the figure (bold box) relative to the Texas Panhandle and Amarillo, TX (star).

between the low- and midlevel mesocyclones (Dowell and Bluestein 2002a,b; Marquis et al. 2012). A description of the data collection platforms and methodology will be presented in section 2, followed by observations of the storm evolution in section 3. Discussion of the relevance of the findings in regard to the current conceptual understanding of RFD surges and low-level mesocyclogenesis will be provided in section 4 , with conclusions and recommendations for future work in section 5 .

\section{Analysis methods}

\section{a. Definition of an internal RFD momentum surge}

Internal RFD momentum surges are analyzed within this study as regions that meet the definition of Lee et al. (2012, p. 3420) as " a distinct acceleration of the outflow within a previously established RFD." As a result, RFD surge boundaries in this study have been analyzed at the leading edge of a region of acceleration in TTUKa radial velocity or dual-Doppler wind speed data. The requirement of a minimum acceleration threshold in order to be considered an internal RFD momentum surge as in Lee et al. (2012) is complicated by the spatial and temporal variability of acceleration across RFD surges in mobile radar data (Fig. 2; see Fig. 1 in supplemental material). However, it is noted that each internal RFD momentum surge analyzed herein has a maximum change in wind speed across the analyzed RFD surge boundary greater than the $13 \mathrm{~m} \mathrm{~s}^{-1}$ threshold applied by Lee et al. (2012).

\section{b. Radar platforms}

The near-surface ${ }^{1}$ wind field during the period of interest (2250-2305) was well sampled by the two TTUKa mobile Doppler radars. The $35-\mathrm{GHz}$ TTUKa radars were designed to sample boundary layer phenomena with high spatial resolution and clear-air sensitivity. During VORTEX2, the radars employed a narrow $\left(0.49^{\circ}\right)$ beamwidth, which resulted in azimuthal spacing of $42.7,85.5$, and $171.0 \mathrm{~m}$ at ranges of 5,10 , and $20 \mathrm{~km}$, respectively. In addition, the TTUKa platforms utilize

\footnotetext{
${ }^{1}$ In the present study, near surface refers to data collected either by the mobile mesonet or the TTUKa radars at a $0.0^{\circ}$-elevation angle, which corresponds to approximately the lowest $80 \mathrm{~m}$ above ground level at ranges considered for this study (Fig. 2).
} 
SMART-R-1

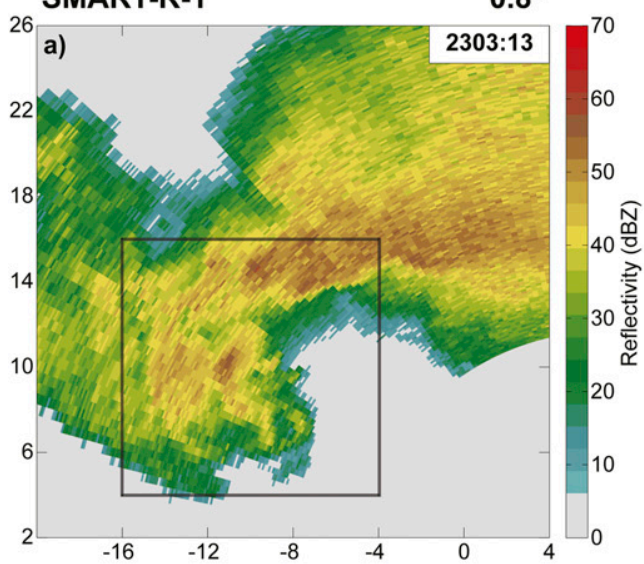

DOW-7

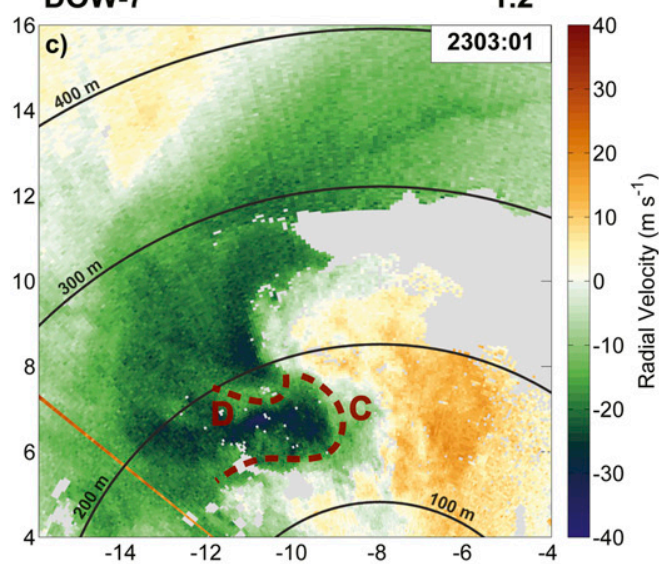

TTUKa-1

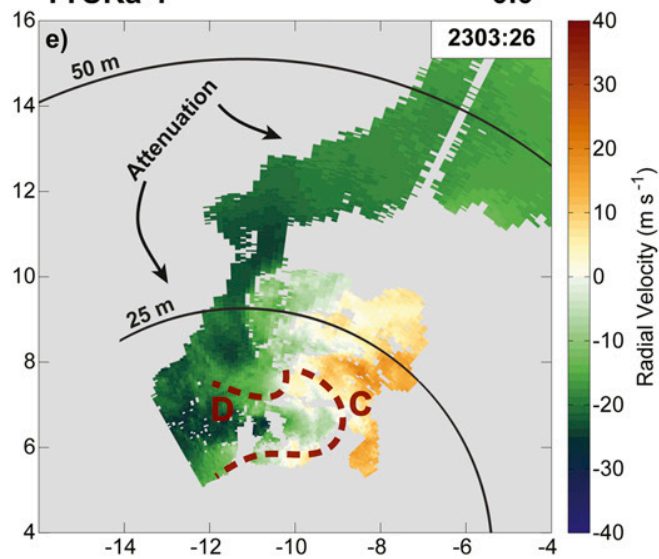

SMART-R-1

$0.8^{\circ}$

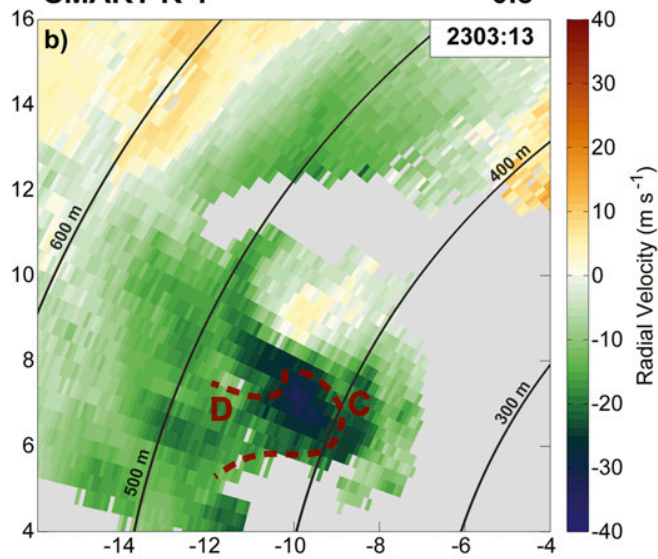

\section{MWR-05XP}

$1.0^{\circ}$
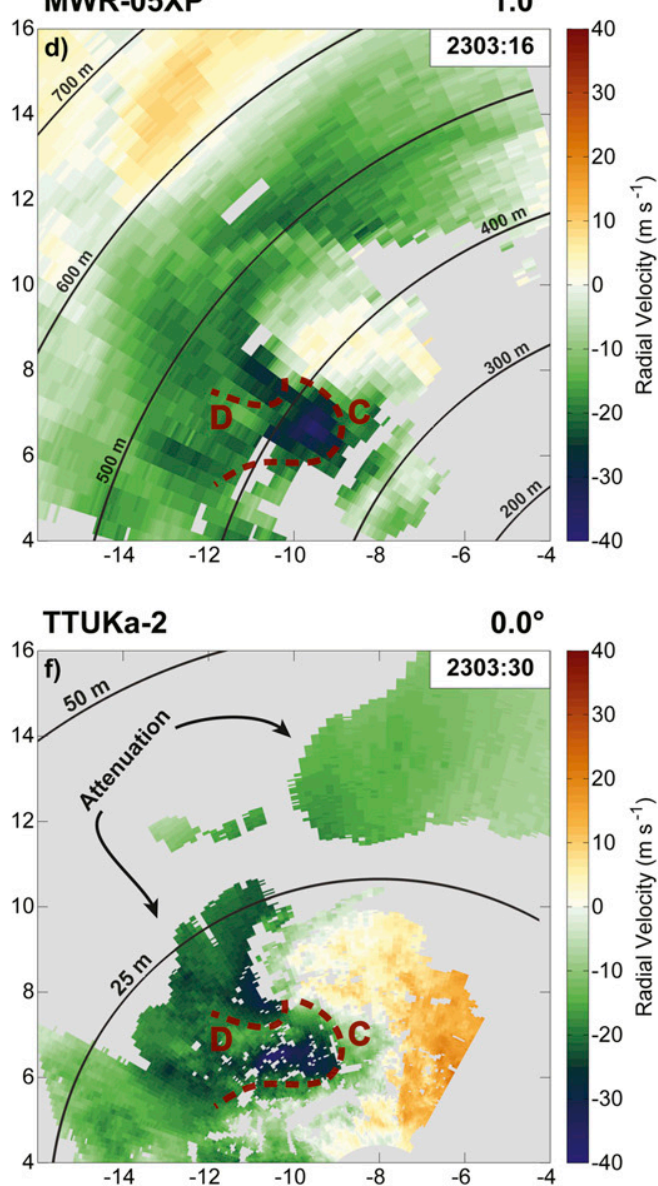

FIG. 2. (a)-(f) Comparison of the low-level radial velocity $\left(\mathrm{m} \mathrm{s}^{-1}\right)$ observations from each available mobile Doppler radar during the period of interest. SMART-R 1 radar reflectivity (dBZ) at 2303:13 and a $0.8^{\circ}$-elevation angle are provided for reference in (a) with the domains in (b)-(f) indicated by the black box. Radial velocity values are plotted from (b) SMART-R 1, (c) DOW-7, (d) MWR-05XP, (e) TTUKa-1, and (f) TTUKa-2 at times (elevation angles) 2303:13 $\left(0.8^{\circ}\right), 2303: 01\left(1.2^{\circ}\right), 2303: 16\left(1.0^{\circ}\right), 2303: 26\left(0.0^{\circ}\right)$, and 2303:30 $\left(0.0^{\circ}\right)$, respectively. Internal RFD momentum surge boundaries subjectively analyzed from TTUKa-2 radial velocity data are indicated by the dashed red lines labeled $\mathrm{C}$ and $\mathrm{D}$ and plotted in an identical location in (b)-(f) to facilitate comparison between platforms. Center-beam heights neglecting the effects of earth curvature are denoted with black circular contours and labeled (m) above the deployment position of TTUKa-1 and regions of missing data are shaded gray. 
TABLE 1. Summary of objective analysis parameters with $R_{\max }$ representing the maximum range, $\kappa$ the Barnes smoothing parameter, $\gamma$ the second-pass convergence parameter, $\Delta$ the grid spacing, and a radius of influence of $(5 \kappa)^{1 / 2}$.

\begin{tabular}{|c|c|c|c|c|c|c|}
\hline Radars & Domain $(x \mathrm{~km} \times y \mathrm{~km} \times z \mathrm{~km})$ & $R_{\max }(\mathrm{km})$ & $\kappa$ & $\gamma$ & $\Delta(\mathrm{m})$ & Radius of influence (m) \\
\hline MWR-05XP & $20 \times 20 \times 12.5$ & 20 & 0.996 & 0.3 & 250 & 2232 \\
\hline TTUKa single Doppler & $20 \times 20$ & 18 & 0.042 & 0.3 & 50 & 458 \\
\hline TTUKa dual Doppler & $7 \times 7$ & 6.8 & 0.0064 & 0.3 & 25 & 179 \\
\hline TTUKa dual Doppler (smooth) & $7 \times 7$ & 6.8 & 0.996 & 0.3 & 25 & 2232 \\
\hline
\end{tabular}

nonlinear frequency modulation pulse compression techniques that allow for fine along-beam resolution (approximately $35 \mathrm{~m}$ ) to be maintained while using a long pulse width $(20 \mu$ s in VORTEX2) for enhanced clear-air sensitivity. A complete description of TTUKa specifications for VORTEX2 is available in Weiss et al. (2009).

The TTUKa radars deployed along a short $(3.3 \mathrm{~km})$ baseline west of Dumas, Texas, from 2242 to 2323, collecting 12 volumes of level, near-synchronous dualDoppler data at elevation angles of $0.0^{\circ}, 0.25^{\circ}$, and $0.5^{\circ}$ between 2256 and 2306. Prior to objective analysis and dual-Doppler synthesis, radar data were reoriented to Earth-relative coordinates by matching ground clutter patterns to known locations of man-made structures, such as power poles and tall towers (Wurman and Gill 2000). Radial velocity data were then thresholded on low values of radar reflectivity factor to remove areas of incoherent radial velocity, and manually edited to remove ground clutter and correct regions of aliased velocities. A final quality control method involved correcting areas of speckling introduced by the interleaved dual pulse repetition frequency (PRF) utilized by the TTUKa radars during VORTEX2 (Jorgensen et al. 2000). Speckling occurred primarily in gates within strong horizontal wind shear where the interleaved velocities were improperly dealiased. New dealiased velocities for speckles were found based on both available maximum unambiguous velocities, with data being retained if falling within an acceptable limit compared to the mean radial velocity of surrounding gates (C. Alexander 2011, personal communication).

Near-surface TTUKa data are complemented by volumetric single-Doppler data with a $1.8^{\circ}\left(2.0^{\circ}\right)$ azimuthal (vertical) beamwidth and a range sampling interval of $75 \mathrm{~m}$ collected by the MWR-05XP radar [complete specifications are available in Bluestein et al. (2010)]. The MWR-05XP utilizes a combination of mechanical and electronic beam steering that allowed a volumetric sector scan with 26 elevation angles $\left(1^{\circ}-\right.$ $40^{\circ}$ ) to be collected in approximately $10 \mathrm{~s}$ for scanning strategies used in VORTEX2. Continuous data collected by the MWR-05XP from 2250 to 2305 provides vertical context for TTUKa observations as well as a greater maximum unambiguous range $(30 \mathrm{~km})$. Quality assurance of MWR-05XP data proceeded similarly to TTUKa data with the exception that despeckling of the data was not necessary. As the MWR-05XP is not equipped with a hydraulic leveling system, additional quality control was necessary to quantify spatial errors in the data and is described in the appendix.

Data from both radar platforms are interpolated to a Cartesian grid using a two-pass Barnes objective analysis scheme (Barnes 1964; Koch et al. 1983; Majcen et al. 2008). Separate analysis domains with varying grid spacing and objective analysis smoothing parameters are used for single-Doppler TTUKa data, dual-Doppler TTUKa data, MWR-05XP data, and "smooth" dualDoppler TTUKa data (Table 1). The domain of each analysis is selected based on the maximum distance to storm features relevant to the analysis $\left(R_{\max }\right)$ in order to minimize the damping of wavelengths retained in the analyses. The Barnes smoothing parameter $(\kappa)$ is defined as $(1.33 \mu)^{2}$ as recommended by Pauley and $\mathrm{Wu}$ (1990), with $\mu$ representing the coarsest gate spacing of data influencing the analysis domain found at a distance of $R_{\max }$ plus the maximum allowable distance between an observation and a grid point $\left[(5 \kappa)^{1 / 2}\right]$ (Trapp and Doswell 2000). A second-pass convergence parameter $(\gamma)$ of 0.3 was employed as in Majcen et al. (2008). Grid spacing $(\Delta)$ in the objective analyses was constrained to be within 0.33 and $0.5 \mu$ (Koch et al. 1983), and extrapolation of data was not permitted.

Dual-Doppler syntheses of ground-relative, objectively analyzed TTUKa radial velocity data were performed for grid points with greater than a $30^{\circ}$ beam-crossing angle collected at a $0.0^{\circ}$-elevation scan. Using $0.0^{\circ}$ elevation scans, coupled with a small difference in elevation $(9 \mathrm{~m})$ between radar deployment sites, allowed the two-dimensional wind field to be calculated without solving for vertical velocity through integration of the mass continuity equation.

Vertical vorticity in objectively analyzed MWR-05XP data was approximated using the azimuthal wind shear (e.g., Byko et al. 2009; Wakimoto et al. (2011); Markowski et al. (2012a)). Azimuthal wind shear was calculated as $\partial V_{r} / \partial \phi\left(r^{-1}\right)$, where $V_{r}$ is the radial velocity, $\phi$ is the azimuth of the radar beam, and $r$ is the range from the 
radar. Though vertical vorticity $(\zeta)$ cannot be retrieved from single-Doppler data, azimuthal wind shear will be equal to $1 / 2 \zeta$ for regions of near-solid-body rotation and a recent study combining single- and dual-Doppler data found that the two fields evolve similarly (Markowski et al. 2012a). A caveat to using azimuthal wind shear as a proxy for vertical vorticity is that it can be influenced by deformation as well as rotation, which can lead to variations in azimuthal wind shear not associated with rotation along low-level gust fronts (Markowski et al. 2012a); however, it is noted that all low-level circulations inferred from azimuthal wind shear presented herein exhibited rotational couplets in MWR-05XP radial velocity data.

\section{c. Mobile mesonet}

The mobile mesonet probes fielded by NSSL and The Pennsylvania State University in VORTEX2 were constructed according to the specifications outlined in Straka et al. (1996) with modifications described by Waugh and Fredrickson (2010). Observations of temperature, relative humidity, pressure, wind speed, and wind direction were collected at $1 \mathrm{~Hz}$ along with time, latitude, and longitude as determined by a global positioning system. As in past studies, wind data during periods of vehicle acceleration were discarded, with the maximum allowable 1-s change in vehicle speed and direction being $2 \mathrm{~m} \mathrm{~s}^{-1}$ and $2^{\circ}$, respectively (Markowski et al. 2012a). Prior to analysis, quality controlled data were smoothed to remove high-frequency noise using a 5-s moving average.

Mobile mesonet data are visualized in two dimensions by applying a time-to-space conversion (Fujita 1955), as described by Markowski et al. (2002). A brief time-tospace conversion window of $180 \mathrm{~s}$ is utilized in this study given the rapid update time between MWR-05XP volumes. The storm motion estimate used for the time-tospace conversion is based on the motion of the leading edge of the first observed internal RFD momentum surge (surge A) in MWR-05XP radial velocity data, as this is the primary feature of interest relative to the mobile mesonet observations. Despite the relatively short time-to-space conversion window, the steadystate assumption necessary for time-to-space conversion is clearly invalid for small-scale features within the Dumas supercell given the rapid evolution apparent in MWR-05XP and TTUKa data (see Figs. 1-4 in supplemental material). It is therefore stressed that the time-to-space conversion analyses within this study represent the approximate storm-relative position of mobile mesonet observations, particularly with respect to rapidly evolving storm features such as internal RFD surges.
Virtual potential temperature, including the effects of liquid water mixing ratio, referred to herein as density potential temperature $\left(\theta_{\rho}\right)$, is calculated in order to assess near-surface buoyancy characteristics sampled by the mobile mesonet. The liquid water mixing ratio is estimated from radar reflectivity values collected by the Shared Mobile Atmospheric Research and Teaching Radar (SMART-R) (Biggerstaff et al. 2005) collected at a $0.8^{\circ}$-elevation angle at $2254: 17,2257: 19,2300: 19$, and 2303:13. Mobile mesonet observations are time-to-space converted relative to the nearest available SMART-R scan, and liquid water mixing ratio is then estimated using the method described by Hane and Ray [1985, their Eq. (6)]. Despite the likely presence of error in the estimation of liquid water mixing ratio resulting from the large amount of precipitation observed within the RFD of the Dumas supercell and height of SMART-R observations above the surface (Figs. 2a,b), the contribution of liquid water mixing ratio to virtual potential temperature was found to be less than $1 \mathrm{~K}$ for all mobile mesonet probes sampling the RFD. Pseudo-equivalent potential temperature (hereafter referred to as equivalent potential temperature, $\theta_{e}$ ) is calculated according to Bolton [1980, his Eqs. (21) and (43)]. As in past mobile mesonet studies, $\theta_{e}$ observations can be compared to similar values on a representative inflow sounding in order to provide qualitative estimates of downdraft origins (Markowski et al. 2002) (Fig. 3c), and can also be utilized to assess the potential buoyancy of near-surface air parcels, as an increase in $\theta_{e}$ is representative of a rightward shift in the air parcel path in a skew $T-\log p$ diagram. Base-state surface values of $\theta_{\rho}$ and $\theta_{e}$ are defined using the average of two 6-min intervals during the period of interest when a mobile mesonet probe traversed a region outside of storm outflow and precipitation, as indicated by TTUKa and MWR-05XP data.

\section{The environment and observations of the Dumas supercell}

\section{a. Storm environment and early evolution}

On the afternoon of 18 May 2010, a shortwave trough moving eastward from the southern Rocky Mountains with up to $20 \mathrm{~m} \mathrm{~s}^{-1}$ southwesterlies at $500 \mathrm{hPa}$ east of the trough axis overspread a moist boundary layer stretching from the Texas Panhandle through eastern Colorado. A radiosonde launched by VORTEX2 $20 \mathrm{~km}$ south of Dumas with a Mobile GPS Advanced Upper Air Sounding System (MGAUS; Parker and Bryan 2010) reveals a favorable environment for supercell development with $0-6-\mathrm{km}$ vertical wind shear greater than $25 \mathrm{~m} \mathrm{~s}^{-1}$ and CAPE values greater than $1500 \mathrm{~J} \mathrm{~kg}^{-1}$ (Fig. 3). A combination of surface heating, deep-layer 


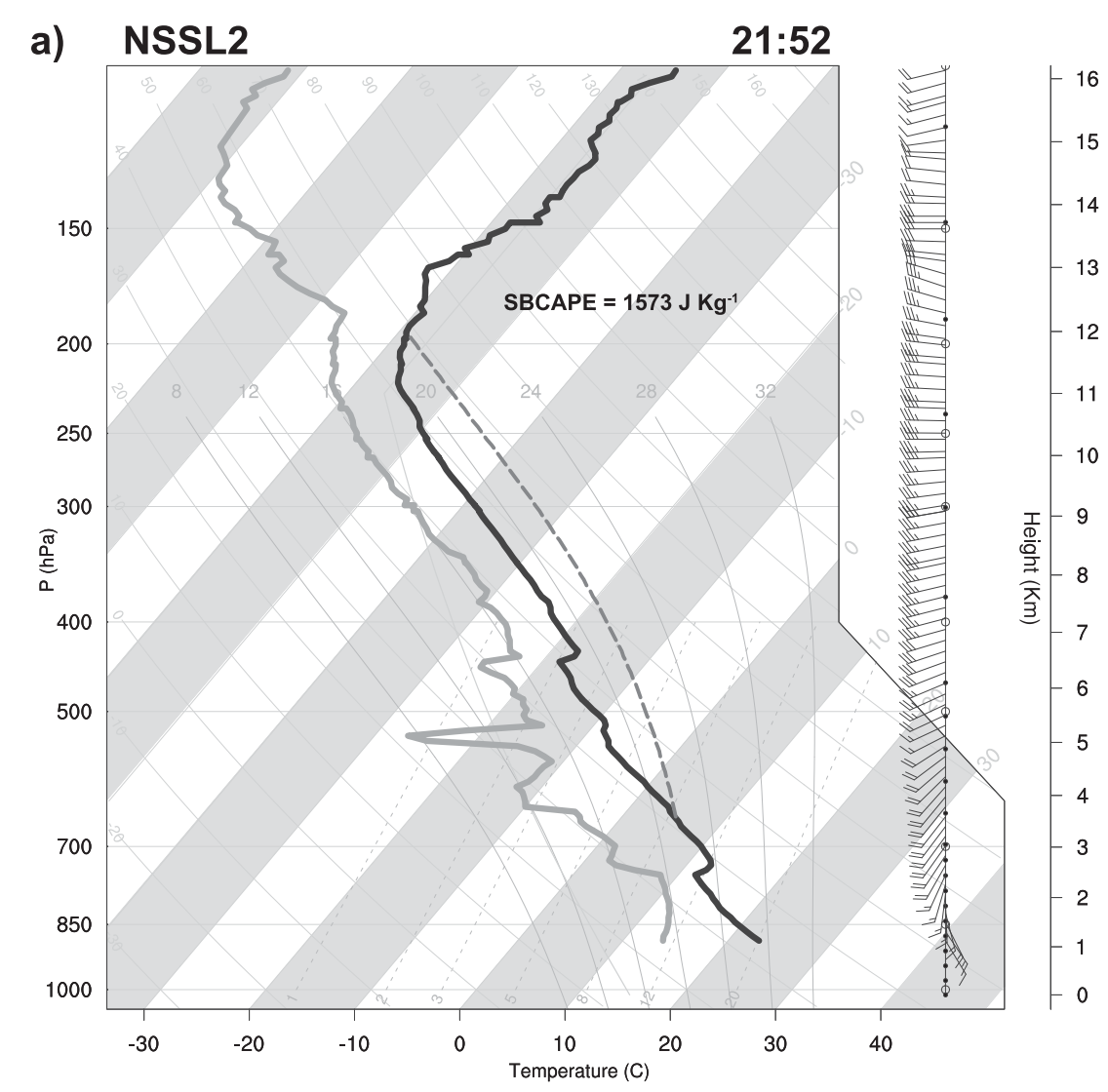

b)
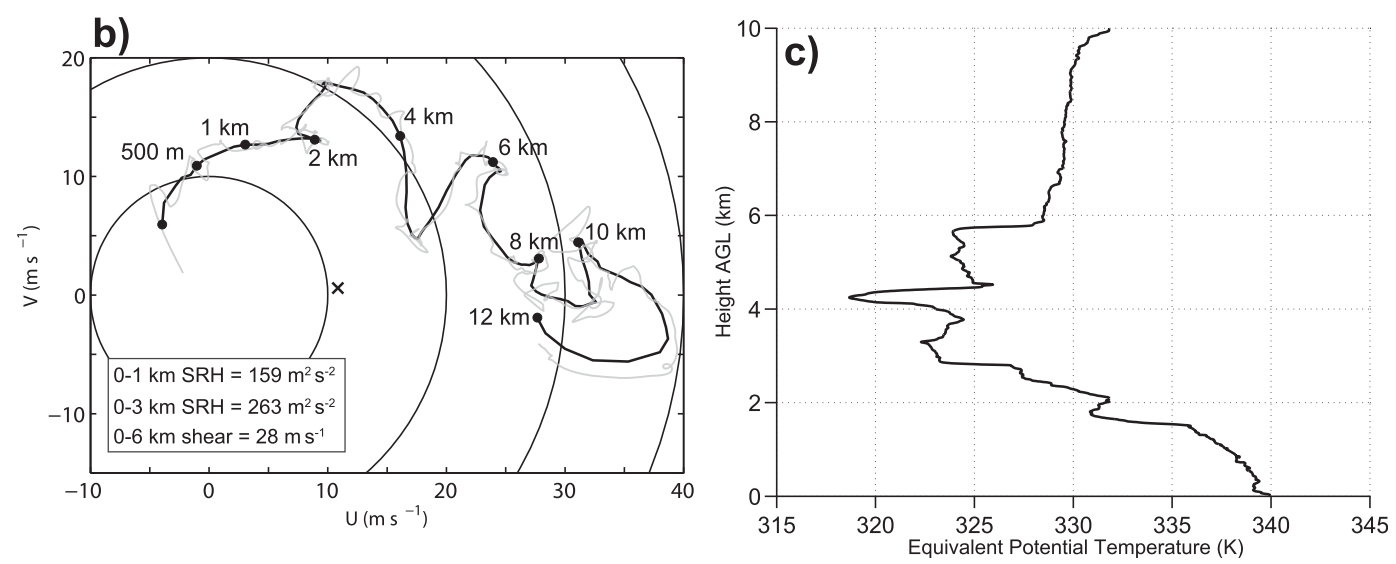

FIG. 3. (a) Skew $T-\log p$ diagram for the 2152 NSSL-2 storm inflow sounding launched from the position indicated in Fig. 1. Environmental virtual temperature is plotted in black, dewpoint temperature in gray, and surface parcel trajectory in dashed gray. (b) Ground-relative hodograph of the 2152 NSSL2 radiosonde; heights above ground level are marked every $2 \mathrm{~km}$, and storm motion is indicated by the $\times$ symbol. Data are smoothed in elevation using a spatial filter of $500 \mathrm{~m}$ and plotted in black with unsmoothed data plotted in gray for reference. (c) Vertical profile of $\theta_{e}$ calculated from the sounding. A five-observation moving average has been applied to the profile to smooth high-frequency fluctuations. Values of surface-based convective available potential energy (SBCAPE), 0-1-km storm-relative helicity (SRH), 0-3-km SRH, and the value of the 0-6-km shear vector are provided in insets to (a) and (b).

ascent ahead of the ejecting shortwave trough and weak convergence along a diffuse dryline aided the initiation of convection by 2100 to the west of Channing, Texas (Fig. 4a). VORTEX2 initially targeted a developing supercell west of Channing at 2130 but refocused on a storm developing to the southwest of the initial target by 2200 as it became the dominant storm (Figs. 4b,c). The target storm underwent a series of three mergers 
with nonsupercellular storms developing to the southwest between 2200 and 2250 (Figs. 4c-f). The last of these ancillary cells merged into the RFD of the target storm between 2240 and 2250 , coincident with the development of a brief tornado west of the primary updraft observed by authors Markowski and Richardson and evident as a strong radial velocity couplet and weakecho reflectivity hole in Doppler on Wheels (DOW) (Wurman et al. 1997) data from 2240 to 2241 (not shown). Following this merger, regions of unorganized convection trailed the target storm and developed into a weaker companion supercell to the west-northwest of the Dumas storm following the period of interest (not shown). As the final merger took place, the visual appearance of the target storm in photos collected by the camera A (CAM A) photogrammetric probe (Wakimoto et al. 2011) (Fig. 1) evolved from a classic supercell with little visible precipitation in the RFD region to a highprecipitation (HP) supercell (Figs. 5a-c). This evolution is similar to recent idealized simulations of storm mergers (Hastings et al. 2012) and observations of classic-to-HP supercell transitions arising from upstream hydrometeor "seeding" by a merging storm (Rasmussen and Straka 1998). By 2250, the target storm exhibited a well-defined, precipitation-filled RFD and a large forward-flank precipitation shield (Fig. 4f).

\section{b. TTUKa observations of the near-surface wind field}

Objectively analyzed radial velocity from TTUKa-2 during the period 2250-2255 reveals an area of enhanced inbound velocity consistent with an internal RFD momentum surge within the broad-scale rear-flank outflow (Fig. 6). Denoted "surge A," this surge has intensified to a distinct region of up to $25 \mathrm{~m} \mathrm{~s}^{-1}$ inbound velocities within much weaker velocities in the broad RFD outflow by 2255:34. The first available TTUKa dual-Doppler synthesis at 2256:13 does not extend westward far enough to capture the approaching surge $\mathrm{A}$, but it does record the passage of the initial RFD gust front (Figs. 7a, 8a). A well-defined convergence zone is present across the RFD gust front, with southeasterly ground-relative winds of $15-20 \mathrm{~m} \mathrm{~s}^{-1}$ ahead of the boundary and southwesterlies generally below $15 \mathrm{~m} \mathrm{~s}^{-1}$ trailing it. Surge $A$ is apparent in the following analysis at 2258:30 as a compact region of up to $40 \mathrm{~m} \mathrm{~s}^{-1}$ southwesterlies extending approximately $3 \mathrm{~km}$ meridionally within the broad RFD (Fig. 7b). It is noted that the RFD gust-front-relative position and areal extent of surge A correspond well to the analyzed position of surge A in single-Doppler data (Fig. 6), which increases confidence that single-Doppler analyses are capturing the pertinent features of the RFD wind field (i.e., the surge location is not significantly influenced by a strong wind component normal to the radar beam).
Maximum winds within surge A decelerate by approximately $15 \mathrm{~m} \mathrm{~s}^{-1}$ over the following $4 \mathrm{~min}$ as the forward motion of the surge slows and it broadens considerably in width (Figs. 7c,d). A second internal RFD surge (surge B) enters the dual-Doppler domain by 2300:02 (Fig. 7c) with two additional surges (surges $C$ and D) rapidly following at 2301:54 (Fig. 7d) and 2302:41 (Fig. 7f), respectively. These surges are additionally apparent as a region of cloud tags accelerating toward the leading edge of the precipitation-filled RFD in video taken by CAM A (Figs. 5e,f). The final three RFD surges exhibit a similar evolution, with convergence along the southern extent and divergence across the apex of each surge. Divergence across the apex of surges B-D (Fig. 8) is unexpected given the rapid acceleration of wind speeds observed with the passage of each surge (Fig. 7). However, it is noted that the "smooth" objective analyses used to calculate the divergence values in Fig. 8 do not resolve narrow bands of convergence apparent across portions of the apex of each surge that are present when the finescale objective analyses are used (not shown), and weak convergence or divergence has been observed across the apex of internal RFD surges in prior dual-Doppler studies (Wurman et al. 2010; Marquis et al. 2012; Kosiba et al. 2013). Surges B-D have a stronger northwesterly component to their wind field and exhibit faster forward movement and higher wind speeds - up to $40 \mathrm{~m} \mathrm{~s}^{-1}$ — than the maturing surge A during this period (Figs. 7e-g). The expansion of the areal extent of the surges with time and their eventual merger (Figs. 7, 8) follows a similar evolution to an internal RFD surge and the broad RFD observed in the 30 April 2000 Crowell, Texas, supercell (Marquis et al. 2008).

A second difference between surge A and surges B-D is that cyclonic curvature in the wind field is consistently observed north of, and trailing, the apex of surges B-D (Figs. 7c-g, 8c-g). This curvature becomes a closed circulation northwest of the northern extent of surge $C$ by 2302:41 (Fig. 7e). Surge D is initially located west of this closed circulation, trailing the apex of surge C (Figs. 7f, $8 \mathrm{f}$ ), and is apparent visually in video and still photographs from CAM A as a developing clear slot wrapping cyclonically around a small wall cloud located within the broad RFD (Figs. 5e,f). Surge D is well defined in dualDoppler synthesis by $2303: 26$ as a narrow region of northwesterlies with velocities up to $40 \mathrm{~m} \mathrm{~s}^{-1}$ just behind its leading edge (Fig. 7f). Additionally at 2303:26, the cyclonic circulation that developed north of the apex of surges $\mathrm{B}$ and $\mathrm{C}$ has further retrograded with respect to the boundary of surge $\mathrm{C}$ and has become located north of the apex of surge $\mathrm{D}$ and collocated with a region of strong convergence (Fig. 8f). A dramatic contraction and intensification of this vortex occurs over the 

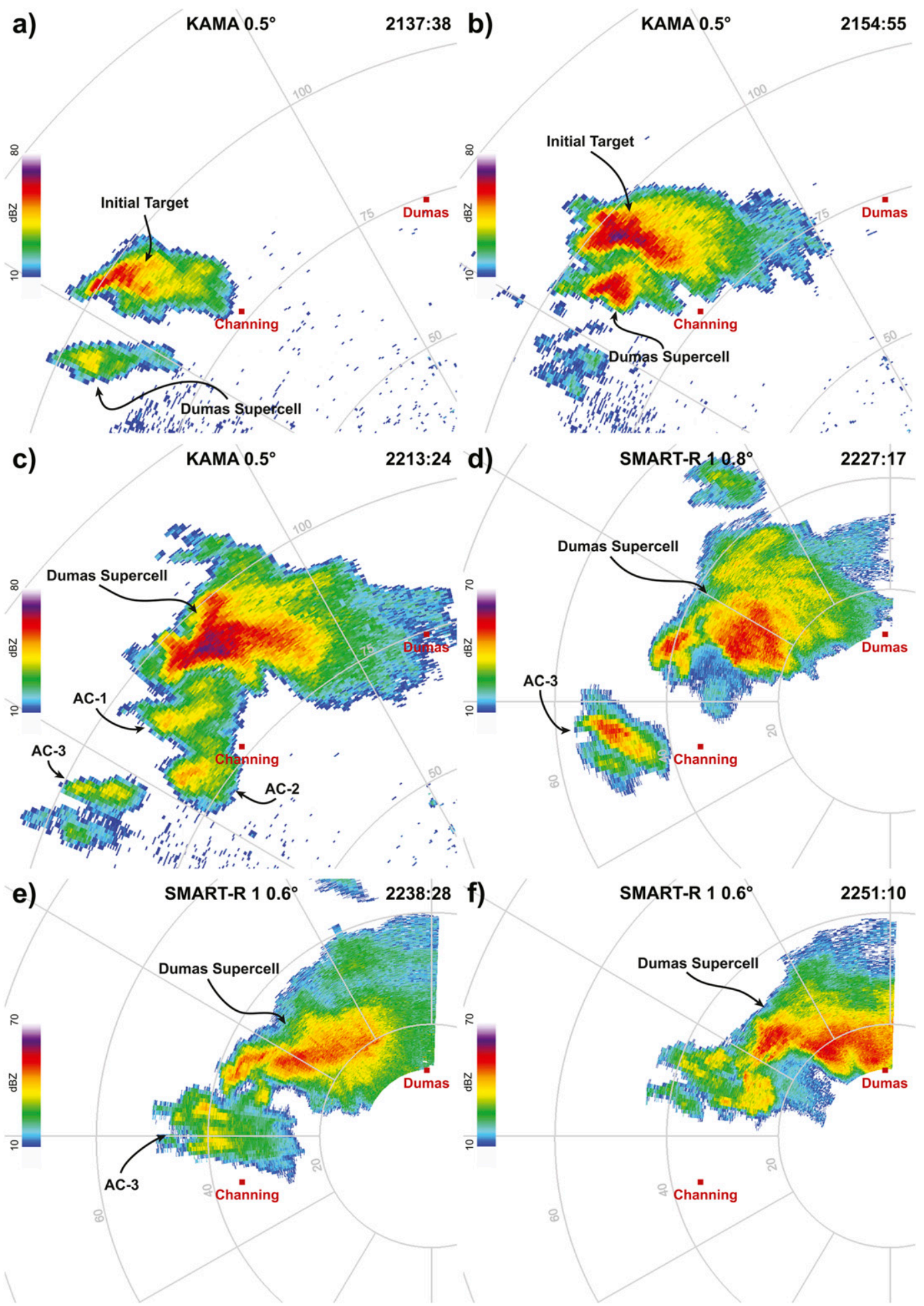

FIG. 4. KAMA $0.5^{\circ}$-elevation angle radar reflectivity (dBZ) observations of the developing Dumas supercell at (a) 2137:38, (b) 2154:55, and (c) 2213:24; and then SMART-R radar reflectivity values at (d) 2227:17, (e) 2238:28, and (f) $2251: 10$ collected at a (d) $0.8^{\circ}$ - and (e),(f) $0.6^{\circ}$-elevation angle. Locations of the initial target storm, Dumas supercell, and three ancillary cells (AC) that merge into the Dumas supercell are annotated. Radar reflectivity values have been thresholded at $10 \mathrm{~dB} Z$ and range rings are given in kilometers. 


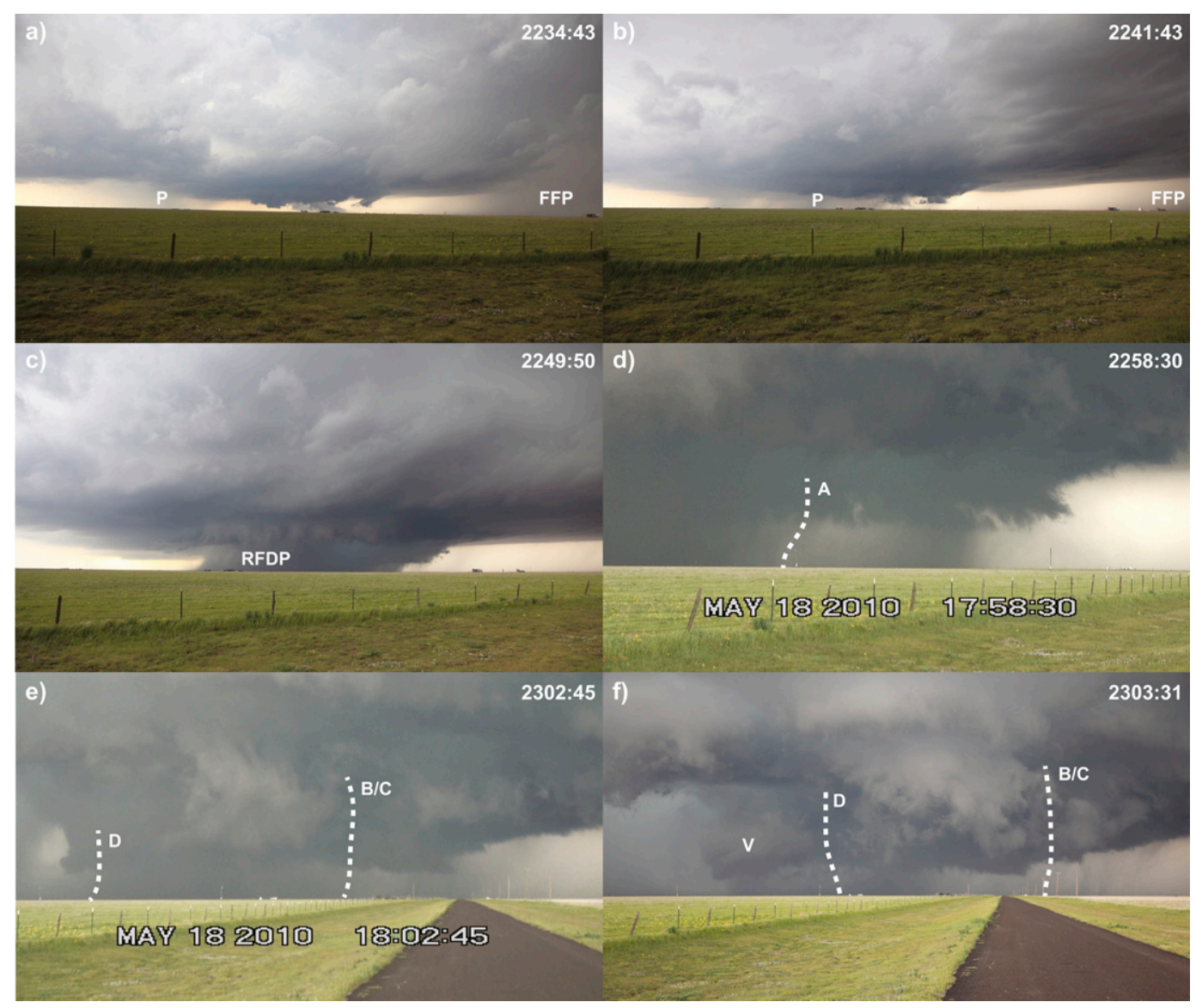

FIG. 5. CAM A still photographs and video captures illustrating the visual evolution of the Dumas supercell at (a) 2234:43, (b) 2241:43, (c) 2249:50, (d) 2258:30, (e) 2302:45, and (f) 2303:31. Rear-flank and forward-flank precipitation in the Dumas supercell are labeled RFDP and FFP, and precipitation from the storm merging into the Dumas supercell is labeled P. Estimated locations of internal RFD surge gust fronts are indicated by dashed white lines as well as labeled A-D for the order in which they were observed; and V denotes the wall cloud associated with the near-surface vortex sampled by the TTUKa radars. Viewing perspective varies from the southwest to the southsouthwest from (a)-(f), with the road visible in (e) and (f) extending to the north.

following $45 \mathrm{~s}$ as it moves along the apex of surge $\mathrm{D}$, and at 2304:13 it exhibits a maximum change in velocity of greater than $70 \mathrm{~m} \mathrm{~s}^{-1}$ across approximately $300 \mathrm{~m}$ (Fig. $7 \mathrm{~g}) .^{2}$ By 2305:00, only a broad, approximately $2-\mathrm{km}$ wide circulation is observed trailing the leading edge of

\footnotetext{
${ }^{2}$ The vortex sampled is collocated with a brief, less than $30 \mathrm{~s}$, period of spiraling reflectivity bands and a weak-echo hole in TTUKa- 1 and TTUKa- 2 data and could be considered a brief, weak tornado. However, this study treats the vortex as nontornadic, as it was not visually identified by any of the personnel participating in VORTEX2 and its existence is likely only known because it was observed by a high-frequency mobile Doppler radar. Similar vortices observed by mobile Doppler radars that straddle the distinction between tornadic and nontornadic have been recently described as "marginal tornadoes" (Wurman and Kosiba 2013) or "sub-tornado-strength convective scale vortices" (Tanamachi et al. 2013).
}

surge $\mathrm{D}$, which has broadened considerably while located in a region of divergence (Fig. 8h), with a deceleration of the winds behind its leading edge and is merging with the remnants of surges A-C (Fig. 7h).

\section{c. MWR-05XP observations}

The 97 volumes of MWR-05XP data collected from 2250 to 2305 reveal rapidly evolving azimuthal wind shear within the low- and midlevel mesocyclones (Figs. 9; see Figs. 2 and 3 in the supplemental material). ${ }^{3}$ During the beginning of the period of interest from

\footnotetext{
${ }^{3}$ Readers are encouraged to view animations of azimuthal wind shear and reflectivity from MWR-05XP volumes in the supplemental material for a more complete representation of the evolution of the low- and midlevel mesocyclones.
} 

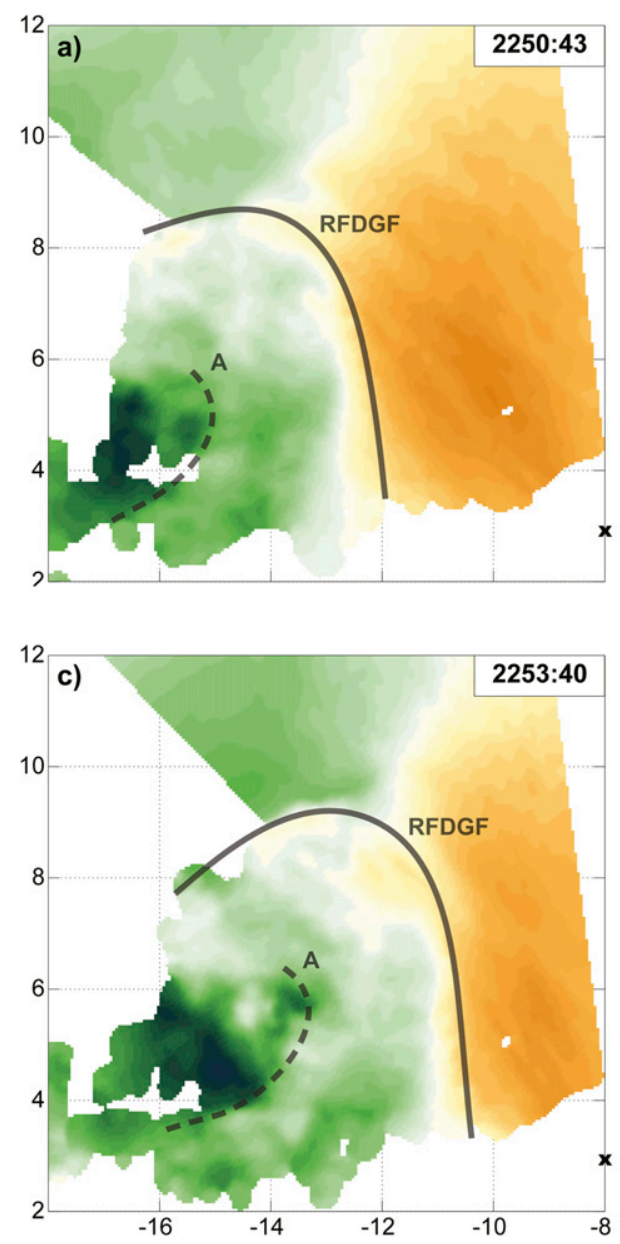
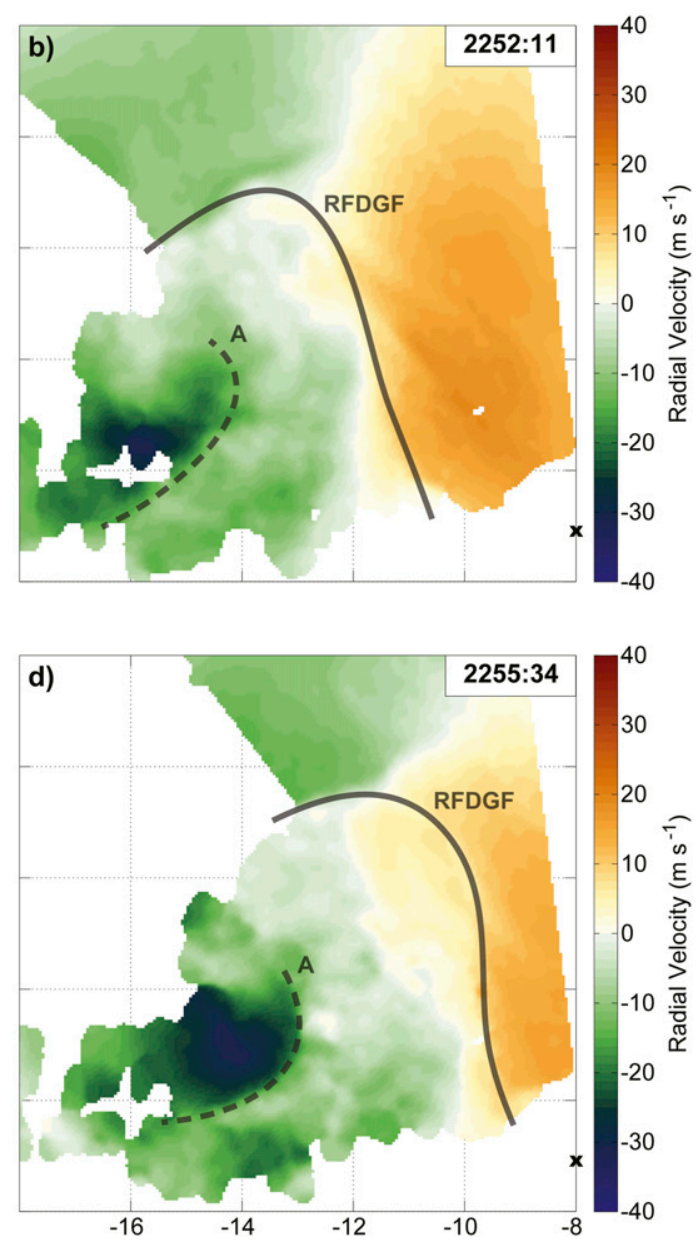

FIG. 6. Objectively analyzed, ground-relative TTUKa-2 $0.0^{\circ}$-elevation radial velocity $\left(\mathrm{m} \mathrm{s}^{-1}\right)$ at (a) $2250: 43$, (b) 2252:11, (c) 2253:40, and (d) 2255:34. Subjectively analyzed location of the primary RFD gust front (bold line) and internal surge A boundary (dashed line) are indicated. The $\times$ symbol represents the location of TTUKa-2, and the grid origin is at the location of the MWR-05XP with axes labeled $(\mathrm{km})$.

approximately 2250 to 2254 , a strong, cyclonic, midlevel mesocyclone with a slight northward tilt and attendant mesoanticyclone is observed (Figs. 9a,b). Though the mesocyclone is apparent to heights greater than $10 \mathrm{~km}$, as sampled by the MWR-05XP, azimuthal wind shear is far weaker below $2 \mathrm{~km}$ above ground level (AGL), indicative of the lack of an intense low-level mesocyclone during this period. Transient regions of cyclonic azimuthal wind shear trailing both the primary mesocyclone and the RFD surges are observed throughout the period of interest and typically are observed for less than $4 \mathrm{~min}$, do not extend below $2.5 \mathrm{~km} \mathrm{AGL}$, and are advected upward with time before dissipating (see Figs. 2 and 3 in the supplemental material). Though observations of vertical velocity are not available within the domain, it is speculated that the transient regions of cyclonic azimuthal wind shear are associated with brief updrafts within the unorganized convection trailing the Dumas supercell during the period of interest (Fig. 4f).

The initially strong midlevel mesocyclone weakens and becomes more strongly tilted with height over the following five minutes, and by 2257 is characterized by a region of cyclonic azimuthal wind shear extending from approximately 2.0 to $7.5 \mathrm{~km}$ AGL (Figs. 9c,d). A region of near-surface cyclonic azimuthal wind shear located above the northern confluence zone of surge A in TTUKa radial velocity and distinct from the midlevel mesocyclone intensifies to values above $0.01 \mathrm{~s}^{-1}$ around 2255 (see Figs. 2 and 3 in the supplemental material). The low-level rotation is initially displaced well to the south and west of the center of the midlevel mesocyclone; however, over the following $5 \mathrm{~min}$ (2255-2300), it slowly moves into a position closer, though still displaced to the south, of the midlevel mesocyclone. During this period the midlevel mesocyclone also 

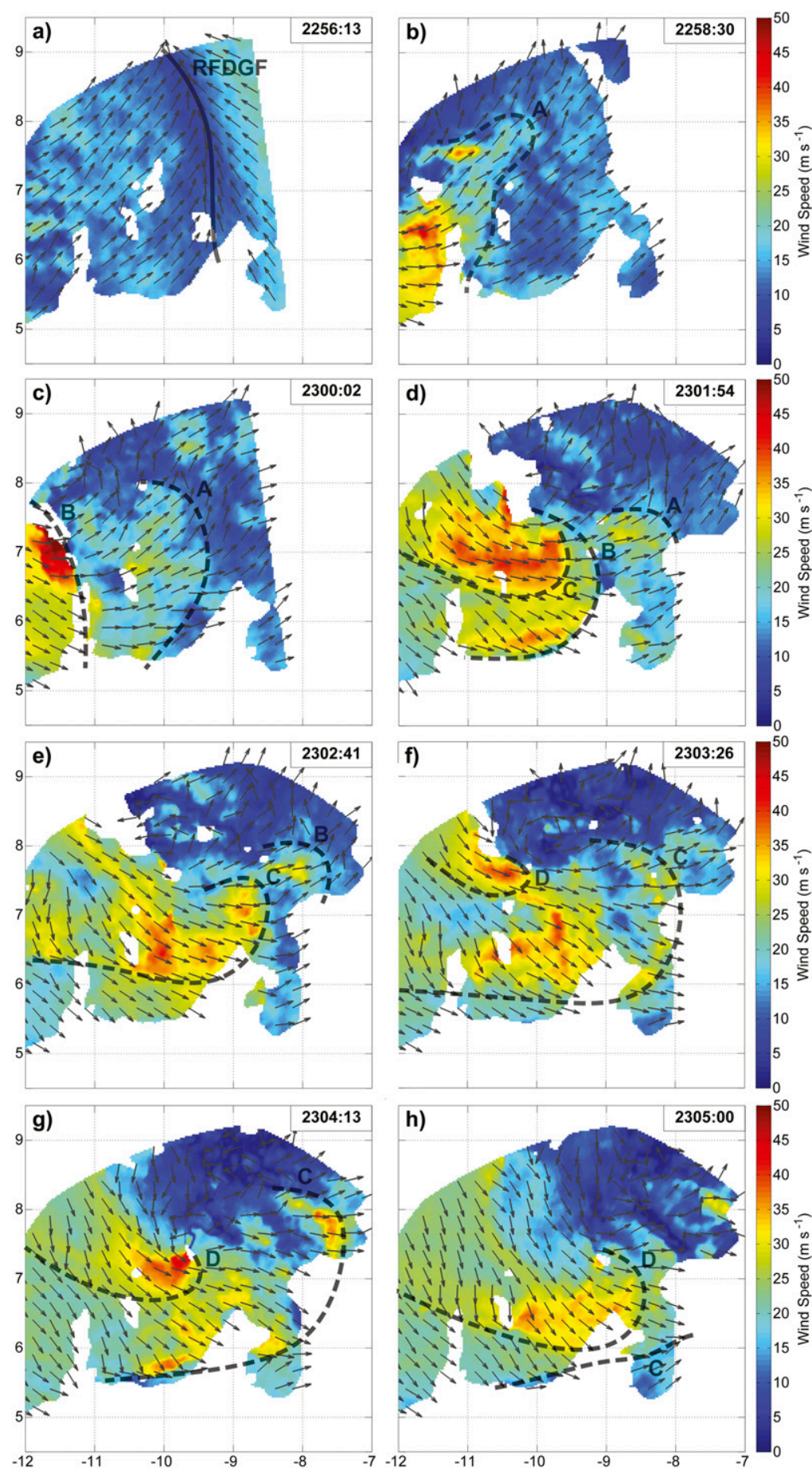

FIG. 7. TTUKa $0.0^{\circ}$-elevation dual-Doppler analyses of ground-relative wind speed (colors, $\mathrm{m} \mathrm{s}^{-1}$ ) and wind direction vectors plotted every eighth grid point at (a) 2256:13, (b) 2258:30, (c) 2300:02, (d) 2301:54, (e) 2302:41, (f) 2303:26, (g) 2304:13, and (h) 2305:00. Subjectively analyzed positions of the primary RFD gust front (RFDGF, bold line) and internal RFD surge boundaries (dashed lines) are indicated. Boundaries analyzed across regions of missing data have been estimated using objectively analyzed TTUKa-2 $0.0^{\circ}$ elevation angle radial velocity data (see Fig. 1 in the supplemental material). Horizontal grid is distance $(\mathrm{km})$ from location of the MWR-05XP. 

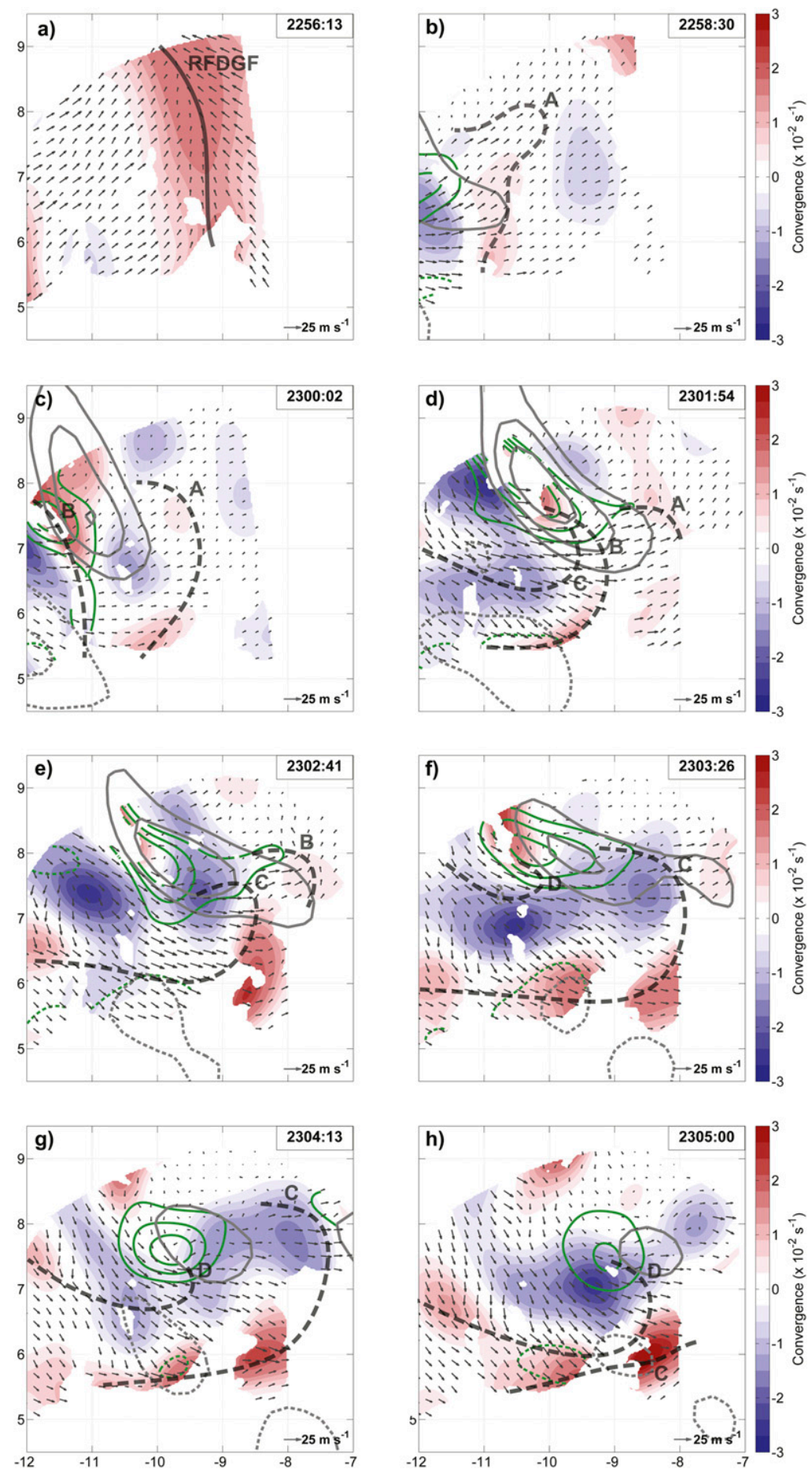

FIG. 8. As in Fig. 7, but for smooth dual-Doppler analyses of convergence $\left(\mathrm{s}^{-1}\right)$ and vertical vorticity $\left(\mathrm{s}^{-1}\right)$. Convergence values are color shaded, and cyclonic (anticyclonic) vertical vorticities are contoured with solid (dashed) green lines with an interval of $0.01 \mathrm{~s}^{-1}$ beginning at $0.02(-0.01) \mathrm{s}^{-1}$. Objectively analyzed values of cyclonic (anticyclonic) MWR-05XP azimuthal wind shear at $500 \mathrm{~m}$ AGL are additionally contoured using solid (dashed) gray lines with an intervals of $0.005 \mathrm{~s}^{-1}$ beginning at $0.01(-0.005) \mathrm{s}^{-1}$ for comparison. Ground-relative wind vectors are plotted every eighth grid point from the finescale wind speed fields presented in Fig. 7. 
reintensifies; though it does not extend vertically throughout the depth of the sample volume and retains a south-to-north tilt, it does develop a broad region of cyclonic azimuthal wind shear greater than $0.02 \mathrm{~s}^{-1}$ between approximately 2 and $5 \mathrm{~km}$ AGL (Figs. 9e,f). A marked increase in the intensity and vertical depth of the low-level mesocyclone occurs shortly after the intensification of the midlevel mesocyclone and by 2301 azimuthal wind shear greater than $0.02 \mathrm{~s}^{-1}$ in the lowlevel mesocyclone extends above $1 \mathrm{~km}$ AGL. Underlain TTUKa-2 radial velocities reveal that the regions of azimuthal wind shear associated with the low-level mesocyclone/

mesoanticyclone couplet straddle the merging internal RFD surges (Figs. 8, 9).

It is noted that Fig. 9e resembles the early development of a low-level mesocyclone in the 5 June 2009 Goshen County, Wyoming, storm (Markowski et al. 2012a, their Fig. 20a). However, unlike the low-level mesocyclone in the Goshen County storm, which grew upward into the midlevels of the storm in the minutes preceding tornadogenesis, the low-level mesocyclone in the Dumas supercell remains distinct from the midlevel mesocyclone and rapidly weakens from 2302 to 2305 . By 2304:13, the low-level mesocyclone is present only as a shallow area of near-surface azimuthal wind shear greater than $0.01 \mathrm{~s}^{-1}$ (Figs. 9g,h).

The MWR-05XP analysis at 2304:13 shows a weakening low-level mesocyclone, which contrasts with a maximum in low-level vortex strength in TTUKa dual-Doppler data at the same time (Fig. $7 \mathrm{~g}$ ). The TTUKa observations of a rapid contraction of the near-surface vortex over the $45 \mathrm{~s}$ prior to $2304: 13$ suggest that the strongest azimuthal wind shear within the low-level circulation may exist at a scale unresolvable by the MWR-05XP (Fig. 2). This hypothesis is supported by the smooth TTUKa dual-Doppler syntheses of vertical vorticity, which are similar in location, though with a greater maximum value, than contours of twice the cyclonic azimuthal wind shear in MWR-05XP data (Fig. 8f). This finding increases confidence that the lack of intense azimuthal wind shear in MWR-05XP analyses is an artifact of the azimuthal resolution of the MWR-05XP and objective analysis scheme.

A significant weakening and lifting of the midlevel mesocyclone is apparent prior to $2304: 13$, resulting in increased total displacement between the low- and midlevel mesocyclones (Figs. 9g,h; see Figs. 2 and 3 in supplemental material), which has been observed coincident with tornado and low-level mesocyclone decay in previous studies (Dowell and Bluestein 2002b; Marquis et al. 2012).

\section{d. Thermodynamic observations of the RFD}

The majority of mobile mesonet data collection from 2250 to 2305 occurred on three east-west roads, with probes performing transects in a similar storm-relative position (Fig. 10). Two probes remained north of the near-surface circulation throughout the period of interest and observed consistently larger thermodynamic deficits than the remainder of the mesonet, with $\theta_{\rho}$ deficits between 3 and $6 \mathrm{~K}$. Deficits of equivalent potential temperature sampled by the northern probes remain below $6 \mathrm{~K}$, which is indicative of air parcels that have either descended from below the elevated mixed layer and associated rapid decrease in $\theta_{e}$ in the Dumas environment (Fig. 3c) or parcels with larger vertical excursions that have entrained high $\theta_{e}$ air from within the storm updraft (Lee et al. 2012). A notable exception to the relatively steady kinematic and thermodynamic conditions north of the low-level mesocyclone occurs at the end of the observing period. A mobile mesonet probe repositioning to the east crosses a boundary to the northeast of the low-level mesocyclone that cuts through the forward-flank precipitation shield. This boundary exhibits a wind shift from northeasterly to easterly coupled with an increase of $\theta_{\rho}$ and $\theta_{e}$ values of approximately $2 \mathrm{~K}$ across the easternmost portion of the transect (Figs. 10e,f). The location of this boundary and observations of relatively small thermodynamic deficits east of the boundary are similar to recently observed (Romine et al. 2008) and simulated (Beck and Weiss 2013) boundaries within the forward flank of supercells.

Neglecting a probe that remained well south of the Dumas mesocyclone, the remaining three probes sampled the environment ahead of the RFD gust front and regions primarily within the leading edge of the broad RFD. From 2250 to 2253, transects across the RFD gust front observed modest $\theta_{\rho}$ and $\theta_{e}$ deficits between 1 and $2 \mathrm{~K}$ within the leading edge of the RFD and southwesterly winds of $10-15 \mathrm{~m} \mathrm{~s}^{-1}$ (not shown), which are similar to the kinematic values produced by dualDoppler analysis at 2256 (Fig. 7a). Over the following 7 min, one probe (Probe 1) performed a north-south transect within the RFD, sampling the environment progressively farther behind the RFD gust front before exiting the RFD to the south (Figs. 10c-f). A time series of Probe 1 observations reveals that $\theta_{\rho}$ and $\theta_{e}$ values within the broad RFD decreased as the probe sampled conditions farther behind the RFD gust front (Fig. 11), which is similar to StickNet (Weiss and Schroeder 2008) observations collected later in the evolution of the Dumas supercell (Weiss et al. 2012) and past findings of thermodynamic heterogeneity within the RFD (Hirth et al. 2008; Lee et al. 2012). Maximum deficits of $\theta_{\rho}\left(\theta_{e}\right)$ 
a)
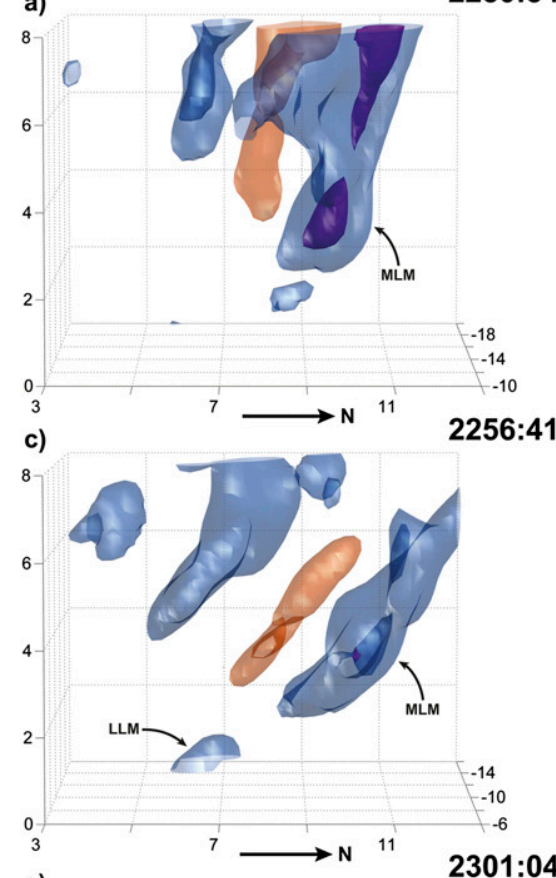

e)

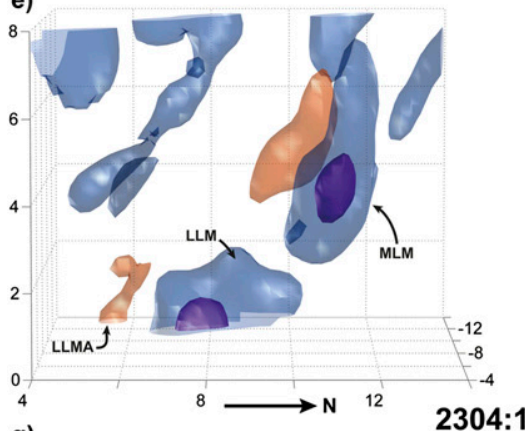

g)

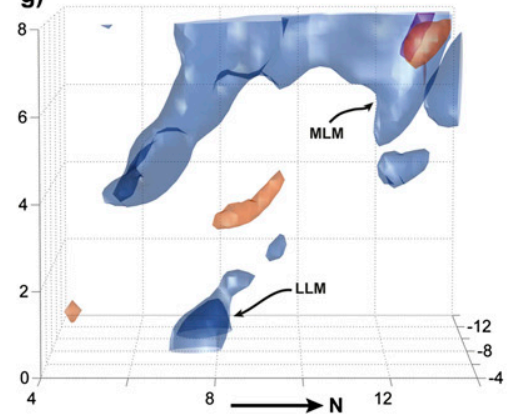

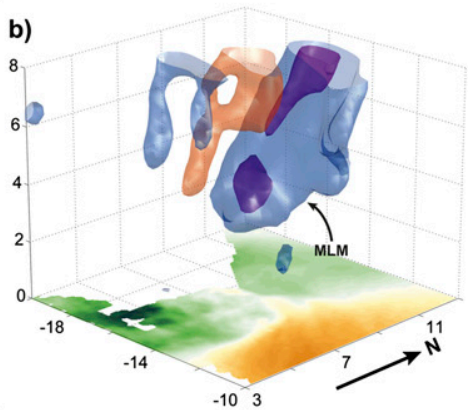
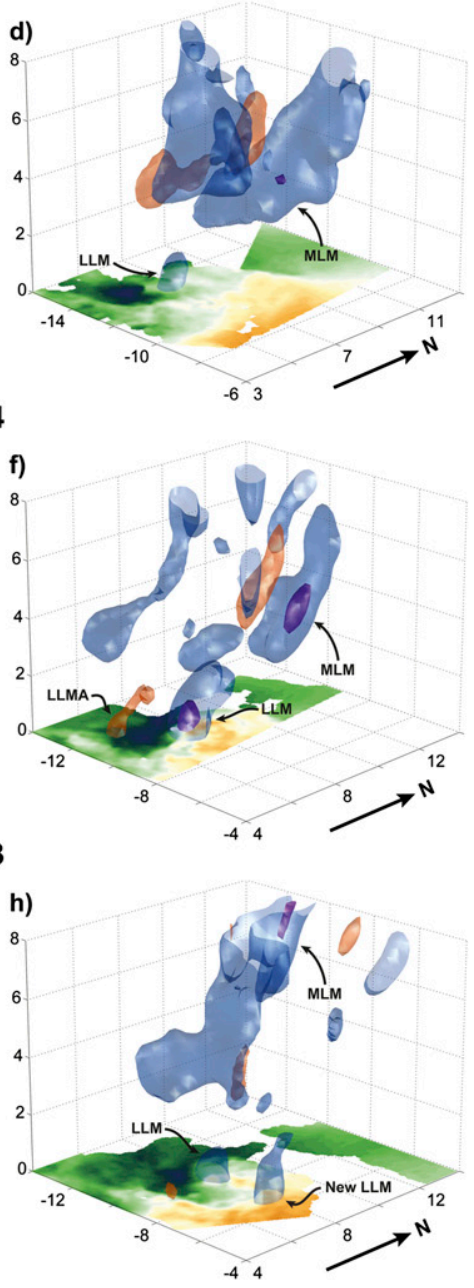

FIG. 9. Three-dimensional isosurfaces of MWR-05XP objectively analyzed azimuthal wind shear $\left(\mathrm{s}^{-1}\right)$ for (a),(b) 2250:34, (c),(d) 2256:41, (e),(f) 2301:04, and (g),(h) 2304:13. Viewing perspectives are (left) from the east at an elevation angle of $6^{\circ}$ and (right) from the southeast at an elevation angle of $20^{\circ}$. Isosurfaces of cyclonic azimuthal wind shear values of $0.01(0.02)$ are plotted in blue (purple), and isosurfaces of anticyclonic azimuthal wind shear values of -0.01 are plotted in orange. (right) Objectively analyzed TTUKa-2 $0.0^{\circ}$ elevation radial velocity $\left(\mathrm{m} \mathrm{s}^{-1}\right)$ at the nearest available time to the MWR-05XP volume is underlain. The times of the TTUKa-2 radial velocity scans match the times of the MWR-05XP volume to within $30 \mathrm{~s}$, and the color table for TTUKa-2 data is the same as in Fig. 6. Regions of cyclonic azimuthal wind shear associated with the low-level (midlevel) mesocyclone are labeled LLM(MLM), anticyclonic azimuthal wind shear associated with the low-level mesoanticyclone is labeled LLMA, and a developing low-level mesocyclone in (h), is denoted New LLM. Grid labels represent distance $(\mathrm{km})$ from MWR-05XP. 
sampled by Probe 1 remain relatively small, below 3 (4) K, which is within ranges typically found within the RFD of tornadic supercells (Markowski et al. 2002; Grzych et al. 2007).

During the southward leg of its transect, Probe 1 briefly traversed the leading edge of surge A (Figs. 10c,d). The time series of observations collected during this transect reveal surge A to have a distinct kinematic and thermodynamic environment from the broad-scale RFD (Fig. 11). Kinematically, the surge is marked by a rapid acceleration of wind speed to values above $20 \mathrm{~m} \mathrm{~s}^{-1}$ and a maximum instantaneous value of $28 \mathrm{~m} \mathrm{~s}^{-1}$. The rapid acceleration of the wind is accompanied by an abrupt warming ${ }^{4}$ of $\theta_{\rho}$ and cooling of $\theta_{e}$ of approximately $1 \mathrm{~K}$. The relative increase of density potential temperature coupled with a decrease in equivalent potential temperature within surge A results from a warmer and drier surge than the broad-scale RFD and is similar to past observations and simulations of RFD surges (Finley and Lee 2004; Lee et al. 2012; Marquis et al. 2012). The relatively warm conditions within surge A during the mobile mesonet transect could arise through either differences in parcel origin height between the RFD surge and broad-scale RFD, changes in the latent chilling within the surge, or some combination of the two. Modification of the latent chilling rate within the RFD surge could be a result of either fewer hydrometeors within the surge or descent in a dynamically driven downdraft that could transport hydrometeor-laden air to the surface rapidly (Kumjian 2011).

\section{Discussion}

\section{a. Evolution of the low- and midlevel mesocyclones}

The initial development of cyclonic azimuthal wind shear associated with the low-level mesocyclone near the surface and observations of counterrotating vortices straddling the leading edge of internal RFD surges throughout the period of interest (Figs. 8, 9) are consistent with the tilting and subsequent stretching of vorticity originating within the RFD surges by upward acceleration. Though calculation of a vorticity budget for air parcels within the RFD surges entering the lowlevel mesocyclone is not possible in the present study, the presence of a vorticity dipole straddling the leading RFD surge is similar to previous studies in which

\footnotetext{
${ }^{4}$ Time lags between the wind acceleration and thermodynamic response as well as discrepancies in the relative rate of change of $\theta_{\rho}$ and $\theta_{e}$ across the boundary are likely attributable to variation in the time constants of mobile mesonet instrumentation (Straka et al. 1996; Waugh and Fredrickson 2010).
}

counterrotating vortices were attributed to upwardarching, baroclinically generated vortex lines (Straka et al. 2007; Markowski et al. 2008, 2011, 2012a; Marquis et al. 2012; Kosiba et al. 2013; Markowski and Richardson 2014) or to downward-sagging, frictionally generated vortex lines (Schenkman et al. 2014).

Evolution of the low-level mesocyclone can be discussed in terms of an inferred dynamic vertical perturbation pressure gradient force, which will be proportional to the magnitude of the azimuthal wind shear if an assumption of pure rotation is made (Rotunno and Klemp 1982). In particular, numerical simulations and observations have found that low-level mesocyclone intensification can be aided by vertical accelerations induced by an upward-directed perturbation pressure gradient force that develops when the lowlevel mesocyclone is minimally displaced horizontally and vertically from an intense midlevel mesocyclone (Wicker and Wilhelmson 1995; Wakimoto et al. 1998; Adlerman et al. 1999; Wakimoto and Cai 2000; Snook and Xue 2008).

Time series of azimuthal wind shear at select vertical levels reveal that changes in near-surface rotation lag changes in azimuthal wind shear in the midlevel mesocyclone at $3 \mathrm{~km}$ (Fig. 12). A pronounced intensification occurs at the low levels after 2259 and follows a decrease in horizontal displacement between the low- and midlevel mesocyclones (Figs. 12c, 13), and during a brief period when a relative maximum in azimuthal wind shear is observed bridging the distinct maxima at low and midlevels (Fig. 13d; see Figs. 2 and 3 in the supplemental material). A reduction in the horizontal displacement between the low- and midlevel mesocyclones would be expected to promote more intense upward-directed perturbation pressure gradient forcing over portions of the low-level mesocyclone, analogous to findings that large horizontal displacement from the maximum midlevel rotation (and associated upward-directed perturbation pressure gradient force) are capable of limiting low-level mesocyclone development (Wicker and Wilhelmson 1995; Snook and Xue 2008) and can occur coincident with tornado and low-level mesocyclone decay (Dowell and Bluestein 2002b; Marquis et al. 2012).

Though smaller than previous times, the horizontal displacement between the maximum azimuthal wind shear at $500 \mathrm{~m}$ and $3 \mathrm{~km}$ during the intensification of the low-level mesocyclone is still greater than $3 \mathrm{~km}$ (Fig. 12c). However, the maximum vertical perturbation pressure gradient may be located away from the axis of minimum perturbation pressure for a tilted mesocyclone (Wakimoto et al. 1998; Wakimoto and Cai 2000), 
2252:57 - 2255:57
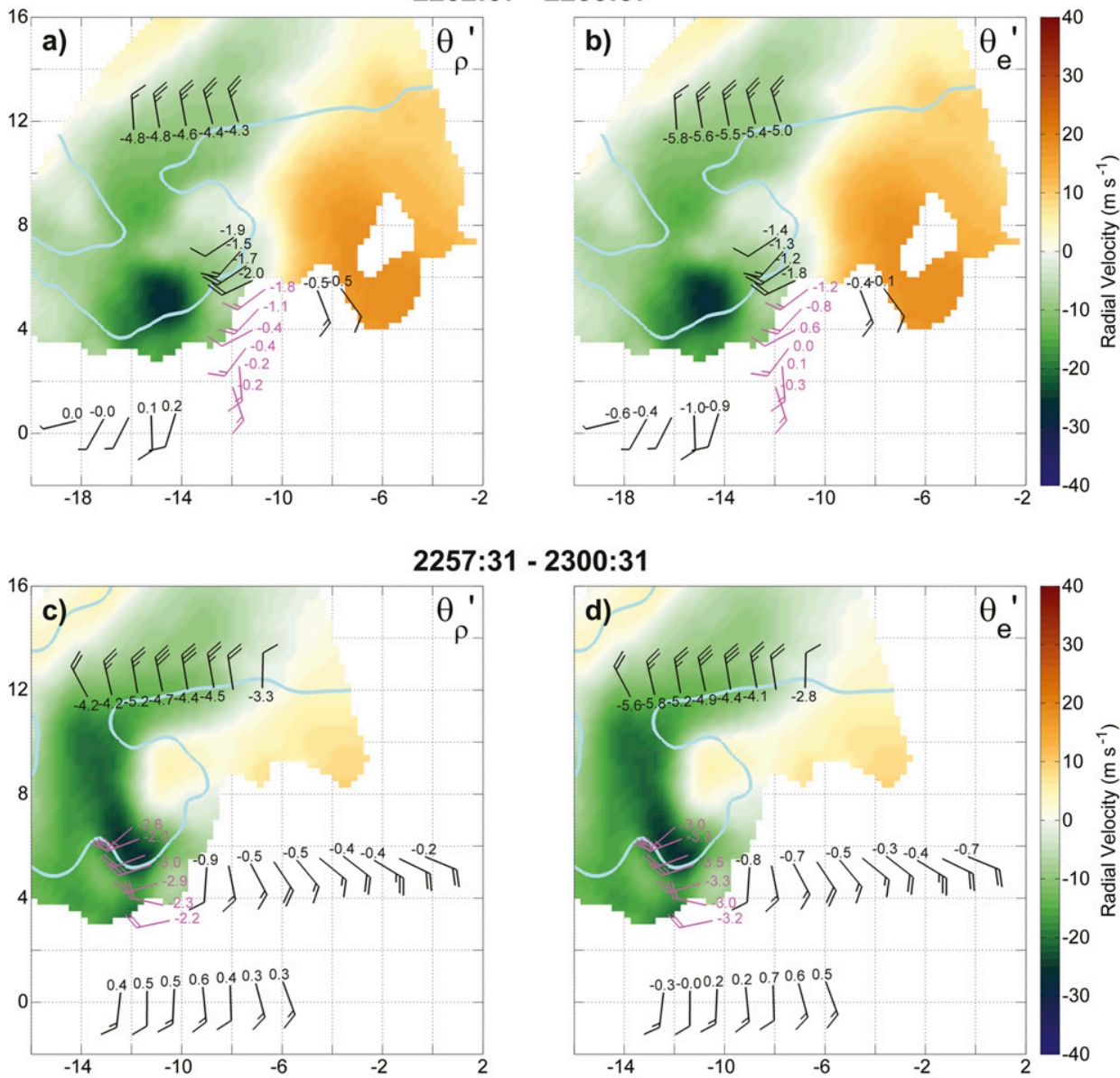

\section{8:56 - 2301:56}
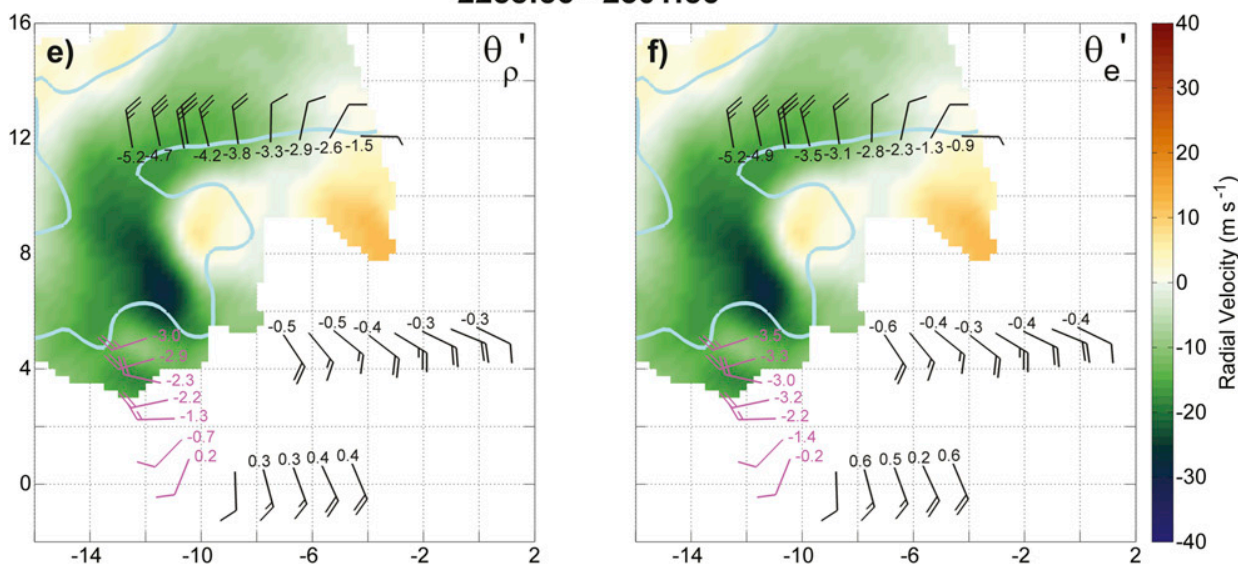

FIG. 10. Time-to-space converted mobile mesonet observations of ground-relative wind speed [half (full) barbs represent 2.5 (5) $\mathrm{m} \mathrm{s}^{-1}$ ] with perturbation (left) $\theta_{\rho}$ and (right) $\theta_{e}$ for 180-s periods centered at (a),(b) 2254:27, (c),(d) 2259:01, and (e),(f) 2300:26. Mobile mesonet observations are plotted every 30 s with selected observations removed for clarity and are overlain on objectively analyzed MWR-05XP $1.0^{\circ}$ elevation radial velocity observations $\left(\mathrm{m} \mathrm{s}^{-1}\right)$. As only a single elevation angle of MWR-05XP data was objectively analyzed here, the height of the analysis will vary across the domain. Observations from Probe 1 are plotted in pink, $30 \mathrm{dBZ}$ MWR-05XP $1.0^{\circ}$ elevation radar reflectivity values are contoured in cyan, and grid labels represent distance $(\mathrm{km})$ from MWR-05XP. 

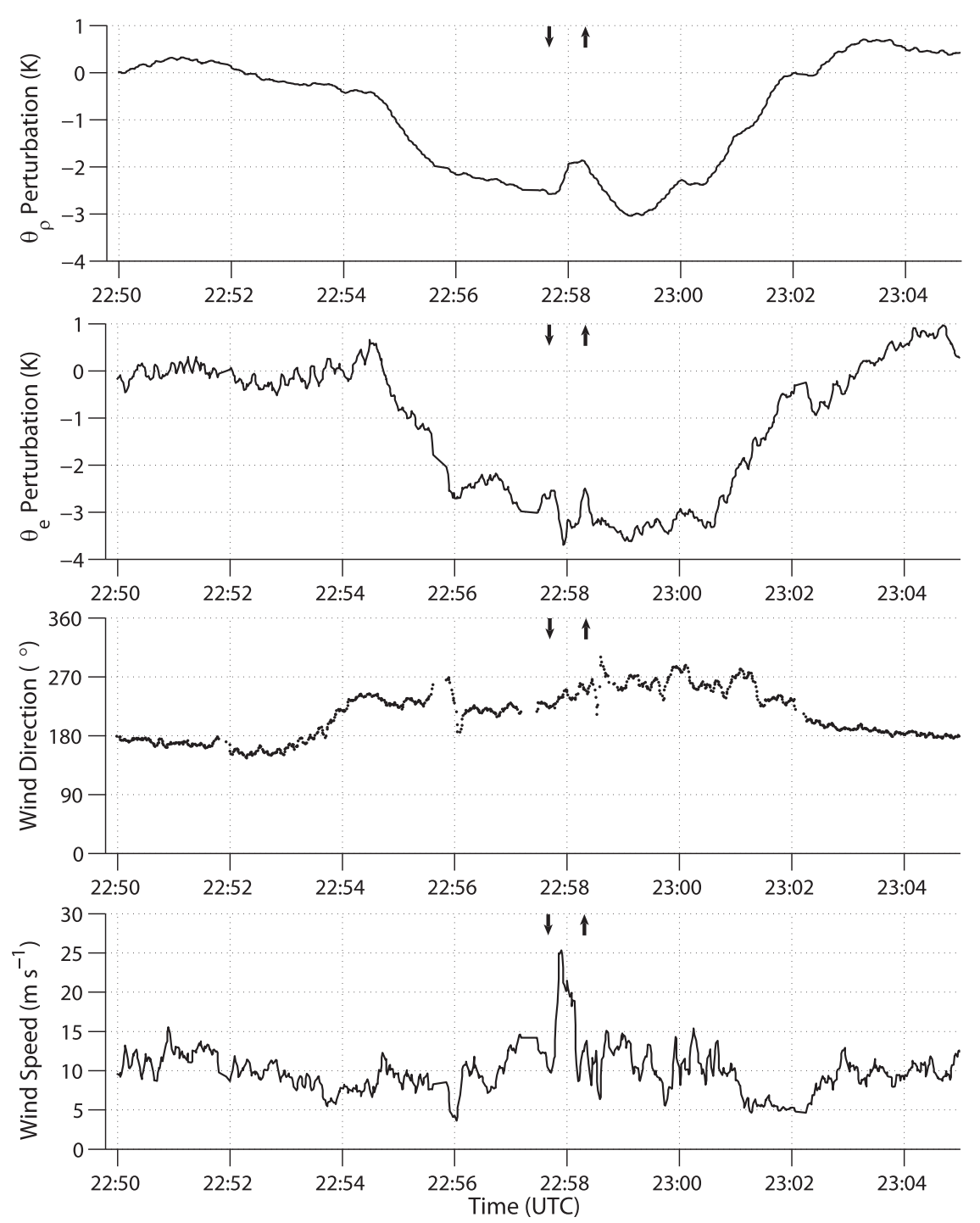

FIG. 11. Time series of Probe 1 observations of (from top to bottom) perturbation $\theta_{\rho}$ and $\theta_{e}$, wind direction, and wind speed. Arrows at the top of each panel represent the times that Probe 1 crossed into (down) and out of (up) internal RFD surge A.

as is observed within the Dumas supercell (Figs. 9, 13; see Figs. 2 and 3 in the supplemental material). Additionally, the relative maximum in azimuthal wind shear bridging the low- and midlevel maxima prior to intensification would result in an increase in azimuthal wind shear with height over portions of the low-level mesocyclone nearest to the midlevel mesocyclone (Figs. 13d; see Figs. 2 and 3 in the supplemental material). These two factors increase confidence that an upward-directed perturbation pressure gradient force is aiding in low-level mesocyclone intensification, despite the horizontal displacement between azimuthal wind shear maxima at $500 \mathrm{~m}$ and $3 \mathrm{~km}$. The rapid decay of the low-level mesocyclone beginning at roughly 2302:30 occurs while it is in a similar midlevel-relative position to when it intensified; however, azimuthal wind shear in the midlevels rapidly weakens and advects upward prior to the decay at low levels (Figs. 9, 12, 13; see Figs. 2 and 3 in the supplemental material), reducing the gradient in azimuthal wind shear with height below the midlevel mesocyclone and implying a decrease in an upwarddirected perturbation pressure gradient force acting on the low levels.

Enhanced near-surface convergence along internal RFD momentum surge boundaries can also generate and maintain low-level upward acceleration and the associated stretching of vertical vorticity (Marquis et al. 2012; Kosiba et al. 2013). It is noted that the maximum in cyclonic azimuthal wind shear at $500 \mathrm{~m}$ in 

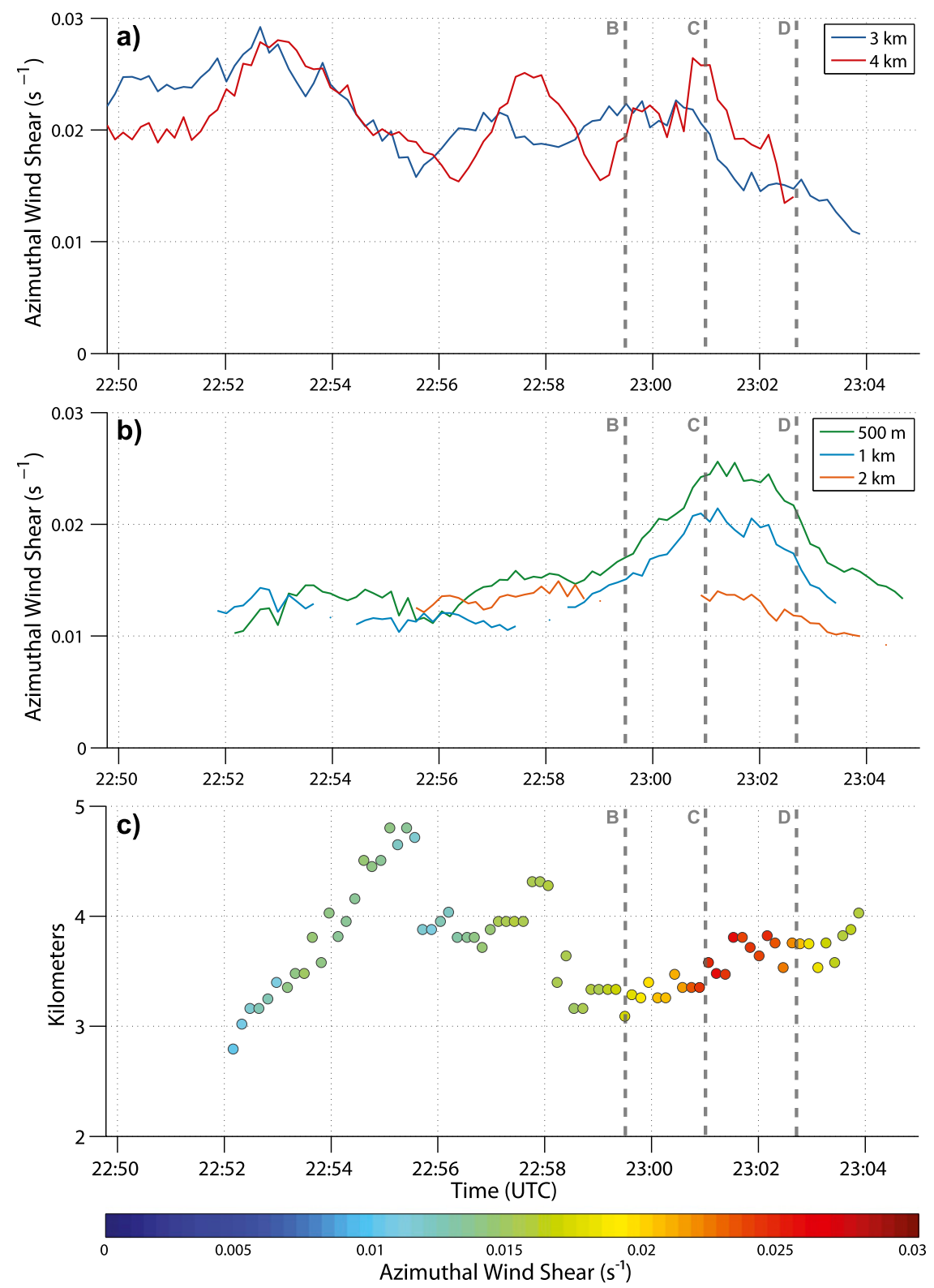

FIG. 12. Time series of maximum cyclonic azimuthal wind shear $\left(\mathrm{s}^{-1}\right)$ in objectively analyzed MWR-05XP data for grid levels: (a) 3 and $4 \mathrm{~km}$, and (b) $500 \mathrm{~m}, 1 \mathrm{~km}$, and $2 \mathrm{~km}$ AGL. (c) Horizontal distance $(\mathrm{km})$ between maximum cyclonic azimuthal wind shear at $3 \mathrm{~km}$ and $500 \mathrm{~m}$. Periods of missing data represent times when the maximum cyclonic azimuthal wind shear at a given level was not associated with the primary low- and midlevel mesocyclones within the Dumas supercell. Data points in (c) are color coded according the maximum cyclonic azimuthal wind shear at $500 \mathrm{~m}$. Vertical dashed lines correspond to the first time that internal surges B-D were observed in TTUKa-2 data.

MWR-05XP data was nearly collocated with the maximum in horizontal convergence north and trailing internal RFD momentum surge boundaries B and C in smoothed TTUKa dual-Doppler data (Figs. 8c-e) before moving ahead of the near-surface convergence zone and becoming located over a region of divergence as the low-level mesocyclone weakened (Figs. 8f-h). This transition of convergence to divergence coincident with the decay of the low-level mesocyclone suggests that the near-surface convergence field is playing a role in the intensification, maintenance, and decay of the low-level mesocyclone. 


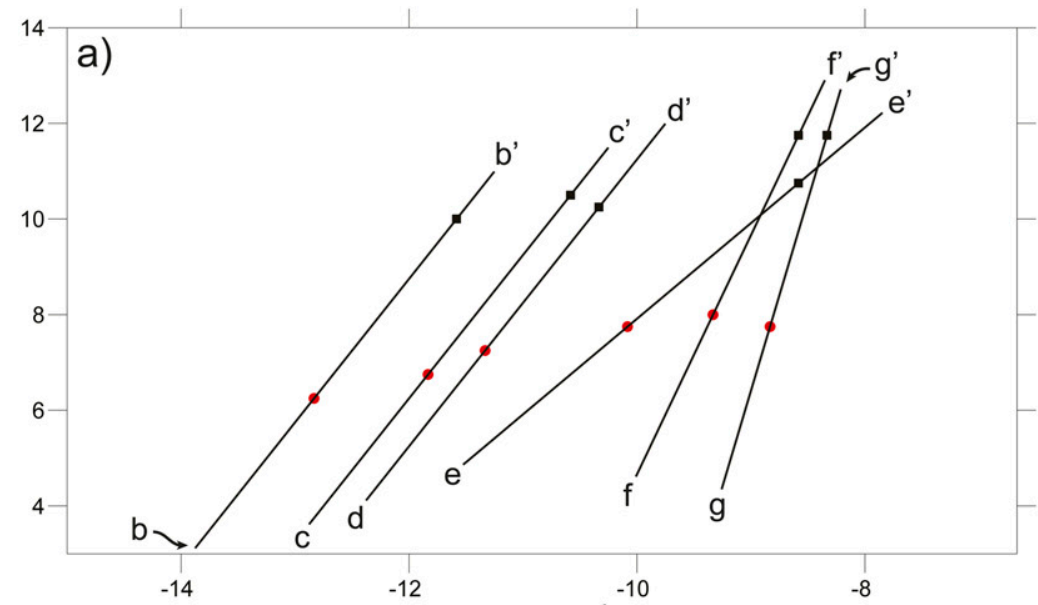

b)

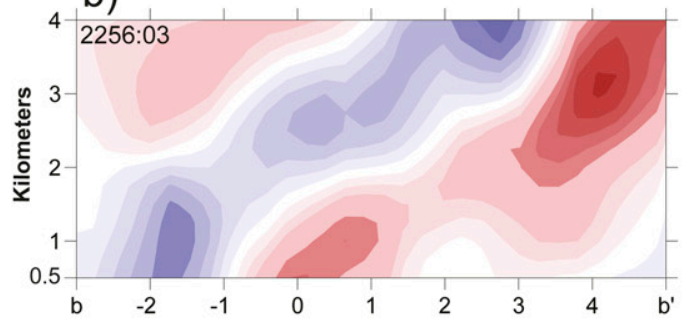

d)

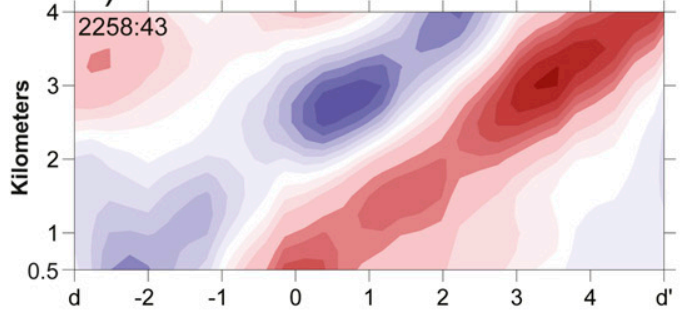

f)

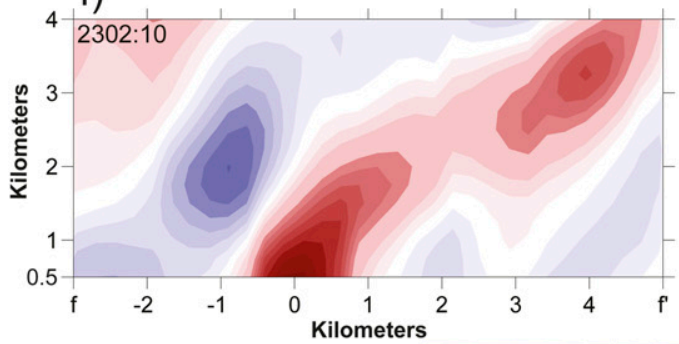

C)

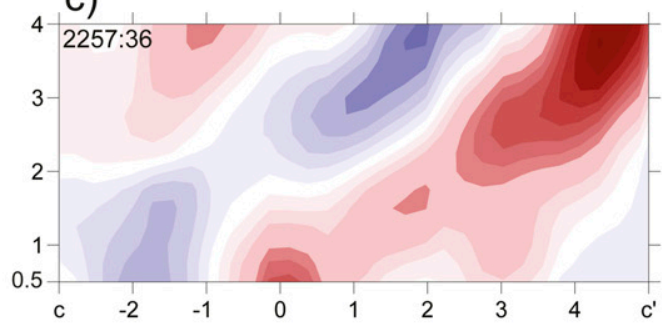

e)

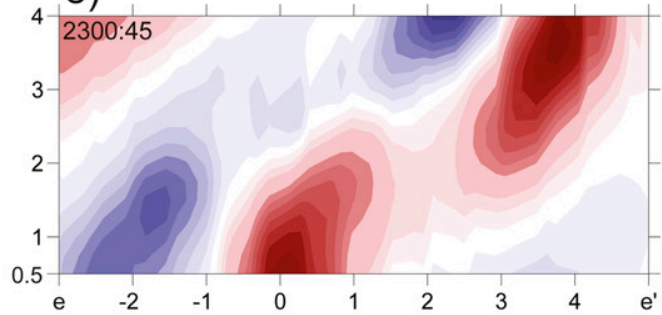

g)

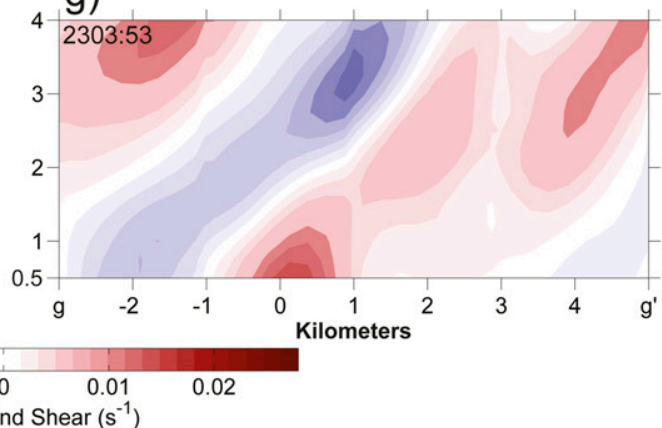

FIG. 13. (a) The locations, relative to the position of the MWR-05XP, of cross-sections of azimuthal wind shear (b)-(g) at (b) 2256:03, (c) 2257:36, (d) 2258:43, (e) 2300:45, (f) 2302:10, and (g) 2303:53. Each cross section is $8 \mathrm{~km}$ in length and passes through the maximum in cyclonic azimuthal wind shear at $500 \mathrm{~m}$ and $3 \mathrm{~km}$ AGL. Labels in (a) correspond to the labels on the horizontal axis of each cross section with the red circles (black squares) in (a) marking the location of the maximum cyclonic azimuthal wind shear at $500 \mathrm{~m}(3 \mathrm{~km})$ AGL. The horizontal axis in (a) has been stretched by a factor of 3 to improve clarity. 
A final note is that upward acceleration and associated enhanced stretching of vertical vorticity is not the only mechanism through which intensification of the low-level mesocyclone can occur. Changes in the buoyancy of air parcels ingested by a low-level mesocyclone will additionally impact the vertical velocity and magnitude of stretching within the mesocyclone (Markowski et al. 2002; Markowski and Richardson 2014).

\section{b. Evolution of the internal RFD surges}

A downward-directed perturbation pressure gradient force within a supercell will occur as vertical vorticity within the low-level mesocyclone intensifies to values greater than those farther aloft, driving an occlusion downdraft (Klemp and Rotunno 1983; Wicker and Wilhelmson 1995; Wakimoto et al. 1998; Adlerman et al. 1999; Wakimoto and Cai 2000). The evolution of surges $\mathrm{B}-\mathrm{D}$ is consistent with the expected behavior of an occlusion downdraft dynamically driven in this manner. Surge B is first observed in TTUKa data just before 2259 along the western periphery of the low-level mesocyclone (see Fig. 1 in the supplemental material), coincident with the rapid increase in azimuthal wind shear in the low levels and following a subtle intensification of azimuthal wind shear at $500 \mathrm{~m}$ AGL to values greater than regions farther aloft (Figs. 7, 12, 13). Furthermore, surge B is initially narrow, exhibits wind speeds higher than the preceding surge A (and far higher than observations of the broad-scale RFD), and wraps cyclonically around the low-level mesocyclone over a period of $4 \mathrm{~min}$ before merging with the remnants of surge A (Figs. 7, 8; see Fig. 1 in the supplemental material), all of which are similar to simulated and observed occlusion downdrafts.

A difference between the evolution of surge B and occlusion downdrafts in numerical modeling and dualDoppler analyses is that occlusion downdrafts have typically been found to develop southeast of the lowlevel mesocyclone (Klemp and Rotunno 1983; Adlerman et al. 1999; Wakimoto and Cai 2000). ${ }^{5}$ However, the minimum vertical velocity within observed and simulated occlusion downdrafts has not occurred along the axis of the minimum perturbation pressure (Klemp and Rotunno 1983; Wakimoto et al. 1998; Wakimoto and Cai 2000). This observation may be attributable to displacement of the maximum downward-directed perturbation pressure gradient force from the axis of minimum perturbation pressure in a tilted mesocyclone (Wakimoto et al. 1998; Wakimoto and Cai 2000) or

\footnotetext{
${ }^{5}$ Though not labeled an occlusion downdraft, a shallow, dynamically driven downdraft was observed south-southwest of the low-level mesocyclone by Wakimoto et al. (2003).
}

a region of updraft surrounding the axis of minimum perturbation pressure, which would result in the minimum vertical velocity occurring at the intersection of the strongest dynamic forcing and minimum "background" vertical velocity along the periphery of the mesocyclone (Markowski 2002). Surges C and D evolve in a similar manner to surge B and are first observed just prior to 2301 and 2303, respectively (Fig. 7), during a time when rotation at low levels was stronger than that aloft (Figs. 12 , 13), suggesting that a downward-directed vertical perturbation pressure gradient force played a role in their development. A final observation on the evolution of surges B-D is that a near-surface vortex developed and persisted to the north and trailing the apex of each surge, reaching a maximum intensity as it interacted with the leading edge of surge D (Figs. 7, 8), which is similar to the evolution of horizontal momentum surges in prior dual-Doppler studies (Marquis et al. 2008; Wurman et al. 2010; Marquis et al. 2012; Kosiba et al. 2013) and numerical simulations (Dahl et al. 2014; Schenkman et al. 2014).

In contrast to observations of internal surges B-D, surge A was first observed and intensified during a period of weak low-level rotation and persisted for more than twice the time either surge $B$ or surge $C$ was observed as a distinct entity within the broad-scale RFD (Figs. 6-8). Observations of slightly warmer conditions within surge A than the surrounding RFD (Figs. 10, 11) suggest that a localized region of enhanced latent chilling is an unlikely driver of the downdraft, though the limited quantity of in situ observations do not rule out regions of stronger thermodynamic forcing trailing the leading edge of the surge (Hirth et al. 2008; Lee et al. 2012; Weiss et al. 2012). Unfortunately, dual-Doppler TTUKa and MWR-05XP data are not available during the genesis of surge $\mathrm{A}$, making a confident assessment of its evolution difficult; it is speculated that the storm merging into the RFD of the Dumas supercell prior to the period of interest may have temporarily enhanced the outflow within the RFD (Hastings et al. 2012), resulting in surge A being a reestablishment of the primary RFD of the Dumas supercell within a region of outflow broadened by the storm merger (Fig. 4).

\section{c. Tornadogenesis failure}

Observations that tornadogenesis is more likely in supercell RFD outflow exhibiting small density potential temperature deficits and considerable potential buoyancy (Markowski et al. 2002; Grzych et al. 2007; Lee et al. 2011, 2012; Markowski et al. 2012a) have been linked to the ability of upward-directed perturbation pressure gradient forces to lift air parcels within the RFD, enhancing the vertical velocity and stretching of 
vertical vorticity within the low-level updraft (Markowski and Richardson 2014). Recent observations of lowlevel mesocyclones in tornadic supercells that grow upward from near the surface to merge with the midlevel mesocyclone and extend through the depth of the troposphere prior to tornadogenesis support this link (Wakimoto et al. 1998; Wakimoto and Cai 2000; Ziegler et al. 2001; Markowski et al. 2012a). It follows that lowlevel mesocyclones whose air parcels are not lifted through the depth of the troposphere would remain distinct from the midlevel mesocyclone, as seen in this study as well as prior dual-Doppler studies (Markowski et al. 2011) and idealized numerical simulations (Markowski and Richardson 2014) of nontornadic supercells. The inability of air parcels within the RFD to be lifted through the depth of the troposphere can be attributed to either excessive negative buoyancy, weak upward-directed perturbation pressure gradient forces due to a weak or displaced midlevel mesocyclone, or a combination of these factors (Markowski and Richardson 2014).

In situ thermodynamic observations within the Dumas supercell revealed $\theta_{\rho}$ deficits within the RFD and surge A to the south of the low-level mesocyclone of generally $2-3 \mathrm{~K}$, which have not been prohibitively large for tornado formation in past studies (Markowski et al. 2002; Grzych et al. 2007). However, limited observations of surge A, a lack of observations within surges B-D, and the possibility of large thermodynamic gradients occurring across successive internal RFD surges (Lee et al. 2012) prevent confident thermodynamic conclusions being made in regard to tornadogenesis failure. The strongest convergence to the north and trailing the northern edge of RFD surges and below the low-level mesocyclone was observed during the development and evolution of surges B and C (Figs. 8c-e, 9) from roughly 2300 to 2303 . The transition from near-surface convergence to divergence underneath the low-level mesocyclone (Figs. 8g,h) coincides with cessation of the inferred upward-directed perturbation pressure gradient force (Figs. 12, 13) and the rapid decay of the low-level mesocyclone. The brevity of near-surface convergence and of a strong low-level mesocyclone coupled with the limited depth of the low-level mesocyclone suggests that upward forcing and stretching of vertical vorticity in parcels originating within the RFD and RFD surges was limited, which could contribute to tornadogenesis failure.

A final observation is that several recent studies have detected descending reflectivity cores (DRCs) prior to tornadogenesis (Rasmussen et al. 2006; Kennedy et al. 2007; Byko et al. 2009; Markowski et al. 2012a), and they have been shown to provide a modulating influence on circulation available to the low-level mesocyclone (Markowski et al. 2012b). It is noted that no DRCs impacting the surface near or within the RFD of the Dumas supercell were observed (see Fig. 4 in the supplemental material).

\section{Summary}

Observations of a low-level mesocyclone cycle and multiple internal RFD surges occurring over a 15 -min period in the 18 May 2010 Dumas, Texas, supercell have been presented. The following behavior was observed within the Dumas supercell:

- Four RFD surges developed and merged over a brief, 15-min period, and an intense surface circulation developed north of the apex of the final RFD surge.

- The second through fourth RFD surges developed in a region of inferred downward-directed perturbation pressure gradient force along the periphery of the low-level mesocyclone and are consistent with the expected surface manifestation of an occlusion downdraft.

- Low-level mesocyclogenesis occurred at the northern extent of an internal RFD surge boundary located entirely within the broad-scale RFD.

- Counterrotating vortices were observed to meridionally straddle the leading edge of RFD surge boundaries throughout the observation period with azimuthal wind shear within the low-level mesocyclone/ mesoanticyclone initially observed near the surface before growing upward, as would be expected with the stretching and advection of vertical vorticity originating in parcels within the RFD surge.

- Intensification and vertical expansion of the lowlevel mesocyclone occurred when it was acted upon by an inferred upward-directed perturbation pressure gradient force associated with a relative minimum in horizontal displacement from the midlevel mesocyclone and above strong near-surface convergence trailing the northern extent of an RFD surge boundary.

- For the majority of the analysis period, the low-level mesocyclone remained distinct from the midlevel mesocyclone and confined to levels below $2 \mathrm{~km}$ AGL, which, combined with moderate buoyancy deficits observed within the RFD, suggest limited vertical acceleration and stretching of vertical vorticity within the low-level mesocyclone, contributing to tornadogenesis failure.

A limitation of this study is the unavailability of volumetric dual-Doppler data required for explicit calculation of thermodynamic and dynamic forcing within the 
Dumas mesocyclones. Efforts are underway to reproduce observed features of the Dumas supercell in ensemble Kalman filter data assimilation experiments. If successful, a quantitative analysis of RFD surge vertical origin and forcing as well as RFD surge impact on low-level mesocyclogenesis and decay can be produced.

Emerging evidence that low-level mesocyclones and tornadic vortex signatures grow upward through the depth of the storm prior to, or during, tornadogenesis (Markowski et al. 2012a; French et al. 2013), coupled with observations of shallow low-level mesocyclones in nontornadic supercells herein and in prior cases (Markowski et al. 2011), suggest that high-temporal resolution studies of low-level mesocyclone depth in tornadic and nontornadic supercells would be a promising research avenue.

Acknowledgments. We are grateful for the dedication of all VORTEX2 participants and in particular Dr. Ivan PopStefanija, Dr. Robert Bluth, Dr. John Schroeder, Jerry Guynes, Scott Gunter, Anthony Reinhart, and Amanda Thibault for their assistance with MWR-05XP and TTUKa data collection. Drs. Matthew Parker, George Bryan, Josh Wurman, Karen Kosiba, Michael Biggerstaff, Louis Wicker, Roger Wakimoto, and Nolan Atkins are thanked for providing mobile sounding, DOW, SMART-R, and photogrammetry data of the Dumas supercell, which were generously hosted by NCAR/EOL under sponsorship of the National Science Foundation. The authors benefited from the SOLO and DORADE Radar Editing algorithms, Detection, Extraction, and Retrieval (DREADER) software as well as additional software provided by Drs. Curtis Alexander, Louis Wicker, Brian Hirth, and Sylvie Lorsolo. We thank Dr. Bruce Lee and two anonymous reviewers for their thoughtful reviews, and Dr. Jim Marquis, Anthony Reinhart, and Daniel Betten for the many helpful conversations during the preparation of this manuscript. This research was supported by NSF Grants AGS-0800542 and AGS-0964088 to Texas Tech University and Grants AGS-0821231 and ATM-0934307 to the University of Oklahoma.

\section{APPENDIX}

\section{Estimation of Spatial Errors in MWR-05XP Data}

The lack of a hydraulic leveling system in the MWR05XP results in spatial errors introduced by offsets in the roll and pitch angles of each radar deployment. Examination of photographs of the MWR-05XP deployment site during the time of interest of the Dumas supercell reveal that there was likely an offset in the roll angle to
TABLE A1. Maximum horizontal and vertical errors in centerbeam location induced by roll angle offsets in MWR-05XP data. Values are for a $5^{\circ}$-elevation angle at a $20-\mathrm{km}$ range, and the effects of earth curvature and refraction have been neglected.

\begin{tabular}{ccc}
\hline \hline $\begin{array}{c}\text { Roll angle } \\
\text { offset }\left(^{\circ}\right)\end{array}$ & $\begin{array}{c}\text { Horizontal displacement } \\
(\mathrm{m})\end{array}$ & $\begin{array}{c}\text { Vertical displacement } \\
(\mathrm{m})\end{array}$ \\
\hline 1 & 30.42 & 347.72 \\
2 & 60.83 & 695.33 \\
3 & 91.23 & 1042.74 \\
\hline
\end{tabular}

the radar deployment with the radar sloping downward from east to west. Despite the presence of potentially significant errors in height in the MWR-05XP data (Table A1), the returned power from scans at a $1^{\circ}$-elevation angle is similar to that at higher angles at all azimuths and ranges, suggesting that the majority of the beam remains above the ground out to the maximum unambiguous range and the roll angle offset is small. Additionally, the locations of storm features (i.e., the low- and midlevel mesocyclones) in MWR-05XP data are approximately collocated with the locations in level SMART-R and DOW data collected at a similar time and elevation angle (Fig. 2), increasing confidence that spatial errors in MWR-05XP data will minimally impact a qualitative analysis of storm features within the Dumas supercell.

\section{REFERENCES}

Adlerman, E. J., 2003: Numerical simulations of cyclic storm behavior: Mesocyclogenesis and tornadogenesis. Ph.D. thesis, University of Oklahoma, $217 \mathrm{pp}$. [Available from School of Meteorology, University of Oklahoma, 120 David L. Boren Blvd., Suite 5900, Norman, OK 73072.]

_, K. K. Droegemeier, and R. Davies-Jones, 1999: A numerical simulation of cyclic mesocyclogenesis. J. Atmos. Sci., 56, 2045-2069, doi:10.1175/1520-0469(1999)056<2045: ANSOCM $>2.0 . \mathrm{CO} ; 2$.

Barnes, S. L., 1964: A technique for maximizing details in numerical weather map analysis. J. Appl. Meteor., 3, 396-409, doi:10.1175/1520-0450(1964)003<0396:ATFMDI >2.0.CO;2.

Beck, J. R., and C. C. Weiss, 2013: An assessment of low-level baroclinity and vorticity within a simulated supercell. Mon. Wea. Rev., 141, 649-669, doi:10.1175/MWR-D-11-00115.1.

Biggerstaff, M. I., and Coauthors, 2005: The Shared Mobile Atmospheric Research and Teaching Radar: A collaboration to enhance research and teaching. Bull. Amer. Meteor. Soc., 86, 1263-1274, doi:10.1175/BAMS-86-9-1263.

Bluestein, H. B., C. C. Weiss, and A. L. Pazmany, 2003: Mobile Doppler observations of a tornado in a supercell near Bassett, Nebraska, on 5 June 1999. Part I: Tornadogenesis. Mon. Wea. Rev., 131, 2954-2967, doi:10.1175/1520-0493(2003)131<2954: MDROOA $>2.0 . \mathrm{CO} ; 2$

, M. M. French, I. PopStefanija, R. T. Bluth, and J. B. Knorr, 2010: A mobile, phased-array Doppler radar for the study of severe convective storms. Bull. Amer. Meteor. Soc., 91, 579600, doi:10.1175/2009BAMS2914.1. 
—, J. B. Houser, M. M. French, J. C. Snyder, G. D. Emmit, I. PopStefanija, C. Baldi, and R. T. Bluth, 2014: Observations of the boundary layer near tornadoes and in supercells using a mobile, collocated, pulsed Doppler lidar and radar. J. Atmos. Oceanic Technol., 31, 302-325, doi:10.1175/ JTECH-D-13-00112.1.

Bolton, D., 1980: The computation of equivalent potential temperature. Mon. Wea. Rev., 108, 1046-1053, doi:10.1175/ 1520-0493(1980)108<1046:TCOEPT>2.0.CO;2.

Byko, Z., P. Markowski, Y. P. Richardson, J. Wurman, and E. Adlerman, 2009: Descending reflectivity cores in supercell thunderstorms observed by mobile radars and in a highresolution numerical simulation. Wea. Forecasting, 24, 155186, doi:10.1175/2008WAF2222116.1.

Dahl, J. M. L., M. D. Parker, and L. J. Wicker, 2014: Imported and storm-generated near-ground vertical vorticity in a simulated supercell. J. Atmos. Sci., doi:10.1175/JAS-D-13-0123.1, in press.

Dowell, D. C., and H. B. Bluestein, 2002a: The 8 June 1995 McLean, Texas, storm. Part I: Observations of cyclic tornadogenesis. Mon. Wea. Rev., 130, 2626-2648, doi:10.1175/ 1520-0493(2002)130<2626:TJMTSP > 2.0.CO;2.

— Part II: Cyclic tornado formation, maintenance, and dissipation. Mon. Wea. Rev., 130, 2649-2670, doi:10.1175/ 1520-0493(2002)130<2649:TJMTSP > 2.0.CO;2.

Finley, C. A., and B. D. Lee, 2004: High resolution mobile mesonet observations of RFD surges in the June 9 Basset, Nebraska supercell during Project ANSWERS 2003. 22nd Conf. on Severe Local Storms, Hyannis, MA, Amer. Meteor. Soc., P11.3. [Available online at https://ams.confex.com/ams/11aram22sls/ techprogram/paper_82005.htm.]

- , and - 2008: Mobile mesonet observations of an intense RFD and multiple RFD gust fronts in the May 23 Quinter, Kansas tornadic supercell during TWISTEX 2008. 24nd Conf. on Severe Local Storms, Savannah, GA, Amer. Meteor. Soc., P3.18. [Available online at https://ams.confex.com/ams/ 24SLS/techprogram/paper_142133.htm.]

French, M. M., H. B. Bluestein, I. PopStefanija, C. Baldi, and R. T. Bluth, 2013: Reexamining the vertical development of tornadic vortex signatures in supercells. Mon. Wea. Rev., 141, 45764601, doi:10.1175/MWR-D-12-00315.1.

Fujita, T. T., 1955: Results of detailed synoptic studies of squall lines. Tellus, 7, 405-436, doi:10.1111/j.2153-3490.1955.tb01181.x.

Grzych, M. L., B. D. Lee, and C. A. Finley, 2007: Thermodynamic analysis of supercell rear-flank downdrafts from Project ANSWERS 2003. Mon. Wea. Rev., 135, 240-246, doi:10.1175/ MWR3288.1.

Hane, C. E., and P. S. Ray, 1985: Pressure and buoyancy fields derived from Doppler radar data in a tornadic thunderstorm. J. Atmos. Sci., 42, 18-35, doi:10.1175/1520-0469(1985)042<0018: PABFDF $>2.0 . \mathrm{CO} ; 2$.

Hastings, R. M., Y. P. Richardson, P. M. Markowski, J. Wurman, and C. C. Weiss, 2012: Mergers in supercell environments. Part I: Conceptual models of mechanisms governing merger outcomes. 26th Conf. on Severe Local Storms, Nashville, TN, Amer. Meteor. Soc., 11B.6. [Available online at https://ams. confex.com/ams/26SLS/webprogram/Paper212519.html.]

Hirth, B. D., J. L. Schroeder, and C. C. Weiss, 2008: Surface analysis of the rear-flank downdraft outflow in two tornadic supercells. Mon. Wea. Rev., 136, 2344-2363, doi:10.1175/ 2007MWR2285.1.

Jorgensen, D. P., T. R. Shepherd, and A. S. Goldstein, 2000: A dual-pulse repetition frequency scheme for mitigating velocity ambiguities of the NOAA P-3 airborne Doppler radar. J. Atmos. Oceanic Technol., 17, 585-594, doi:10.1175/ 1520-0426(2000)017<0585:ADPRFS >2.0.CO;2.

Karstens, C. D., T. M. Samaras, B. D. Lee, W. A. Gallus, and C. A. Finley, 2010: Near-ground pressure and wind measurements in tornadoes. Mon. Wea. Rev., 138, 2570-2588, doi:10.1175/ 2010MWR3201.1.

- W. A. Gallus Jr., B. D. Lee, and C. A. Finley, 2013: Analysis of tornado-induced tree fall using aerial photography from the Joplin, Missouri, and Tuscaloosa-Birmingham, Alabama, tornadoes of 2011. J. Appl. Meteor. Climatol., 52, 1049-1068, doi:10.1175/JAMC-D-12-0206.1.

Kennedy, A. D., J. M. Straka, and E. N. Rasmussen, 2007: A statistical study of the association of DRCs with supercells and tornadoes. Wea. Forecasting, 22, 1191-1199, doi:10.1175/ 2007WAF2006095.1.

Klemp, J. B., and R. Rotunno, 1983: A study of the tornadic region within a supercell thunderstorm. J. Atmos. Sci., 40, 359-377, doi:10.1175/1520-0469(1983)040<0359:ASOTTR>2.0.CO;2.

Koch, S. E., M. desJardins, and P. J. Kocin, 1983: An interactive Barnes objective map analysis scheme for use with satellite and conventional data. J. Climate Appl. Meteor., 22, 1487-1503, doi:10.1175/1520-0450(1983)022<1487:AIBOMA>2.0.CO;2.

Kosiba, K. A., J. Wurman, Y. P. Richardson, P. Markowski, P. Robinson, and J. Marquis, 2013: Genesis of the Goshen County, Wyoming, tornado on 5 June 2009 during VORTEX2. Mon. Wea. Rev., 141,1157-1181, doi:10.1175/MWR-D-12-00056.1.

Kumjian, M. R., 2011: Precipitation properties of supercell hook echoes. Electron. J. Severe Storms Meteor., 6 (5). [Available online at http://ejssm.org/ojs/index.php/ejssm/article/viewArticle/93.]

Lee, B. D., C. A. Finley, and P. S. Skinner, 2004: Thermodynamic and kinematic analysis of multiple RFD surges for the 24 June 2003 Manchester, SD, cyclic tornadic supercell during Project ANSWERS 2003. 22nd Conf. on Severe Local Storms, Hyannis, MA, Amer. Meteor. Soc., P11.2. [Available online at https://ams.confex.com/ams/11aram22sls/techprogram/paper_ 82000.htm.]

,$- \ldots$, and T. M. Samaras, 2011: Surface analysis near and within the Tipton, Kansas, tornado on 29 May 2008. Mon. Wea. Rev., 139, 370-386, doi:10.1175/2010MWR3454.1.

—_ _ _ , and C. D. Karstens, 2012: The Bowdle, South Dakota, cyclic tornadic supercell of 22 May 2010: Surface analysis of rear-flank downdraft evolution and multiple internal surges. Mon. Wea. Rev., 140, 3419-3441, doi:10.1175/ MWR-D-11-00351.1.

Majcen, M., P. Markowski, Y. P. Richardson, D. Dowell, and J. Wurman, 2008: Multipass objective analysis of Doppler radar data. J. Atmos. Oceanic Technol., 25, 1845-1858, doi:10.1175/2008JTECHA1089.1.

Markowski, P. M., 2002: Hook echoes and rear-flank downdrafts: A review. Mon. Wea. Rev., 130, 852-876, doi:10.1175/ 1520-0493(2002)130<0852:HEARFD > 2.0.CO;2.

- and Y. P. Richardson, 2014: The influence of environmental low-level shear and cold pools on tornadogenesis: Insights from idealized simulations. J. Atmos. Sci., 71, 243-275, doi:10.1175/JAS-D-13-0159.1.

— J. M. Straka, and E. N. Rasmussen, 2002: Direct surface thermodynamic measurements within the rear-flank downdrafts of nontornadic and tornadic supercells. Mon. Wea. Rev., 130, 1692-1721, doi:10.1175/1520-0493(2002)130<1692: DSTOWT $>2.0 . \mathrm{CO} ; 2$.

,$--\longrightarrow$, R. P. Davies-Jones, Y. P. Richardson, and J. Trapp, 2008: Vortex lines within low-level mesocyclones 
obtained from pseudo-dual-Doppler radar observations. Mon. Wea. Rev., 136, 3513-3535, doi:10.1175/2008MWR2315.1.

_- M. Majcen, Y. P. Richardson, J. Marquis, and J. Wurman, 2011: Characteristics of the wind field in a trio of nontornadic low-level mesocyclones observed by the Doppler on Wheels radars. Electron. Severe Storms Meteor., 6 (3). [Available online at http://www.ejssm.org/ojs/index.php/ejssm/article/ viewArticle/75.]

_ , and Coauthors, 2012a: The pretornadic phase of the Goshen County, Wyoming, supercell of 5 June 2009 intercepted by VORTEX2. Part I: Evolution of kinematic and surface thermodynamic fields. Mon. Wea. Rev., 140, 2887-2915, doi:10.1175/MWR-D-11-00336.1.

_ and Coauthors, 2012b: The pretornadic phase of the Goshen County, Wyoming, supercell of 5 June 2009 intercepted by VORTEX2. Part II: Intensification of low-level rotation. Mon. Wea. Rev., 140, 2916-2938, doi:10.1175/MWR-D-11-00337.1.

Marquis, J., Y. P. Richardson, J. Wurman, and P. M. Markowski, 2008: Single- and dual-Doppler analysis of a tornadic vortex and surrounding storm-scale flow in the Crowell, Texas, supercell of 30 April 2000. Mon. Wea. Rev., 136, 5017-5043, doi:10.1175/2008MWR2442.1.

,-- , P. M. Markowski, D. C. Dowell, and J. Wurman, 2012: Tornado maintenance investigated with high-resolution dualDoppler and EnKF analysis. Mon. Wea. Rev., 140, 3-27, doi:10.1175/MWR-D-11-00025.1.

Mashiko, W., H. Niino, and T. Kato, 2009: Numerical simulation of tornadogenesis in an outer-rainband minisupercell of Typhoon Shanshan on 17 September 2006. Mon. Wea. Rev., 137, 4238-4260, doi:10.1175/2009MWR2959.1.

Parker, M. D., and G. H. Bryan, 2010: Mobile soundings during VORTEX2: Survey and preliminary results. 25th Conf. on Severe Local Storms, Denver, CO, Amer. Meteor. Soc., 5.6. [Available online at https://ams.confex.com/ams/25SLS/techprogram/ paper_175917.htm.]

Pauley, P. M., and X. Wu, 1990: The theoretical, discrete, and actual response of the Barnes objective analysis scheme for oneand two-dimensional fields. Mon. Wea. Rev., 118, 1145-1164 doi:10.1175/1520-0493(1990)118<1145:TTDAAR>2.0.CO;2.

Rasmussen, E. N., and J. M. Straka, 1998: Variations in supercell morphology. Part I: Observations of the role of upper-level storm-relative flow. Mon. Wea. Rev., 126, 2406-2421, doi:10.1175/ 1520-0493(1998)126<2406:VISMPI >2.0.CO;2.

$\longrightarrow,-$ R. P. Davies-Jones, C. A. Doswell, F. H. Carr, M. D. Eilts, and D. R. MacGorman, 1994: Verification of the Origins of Rotation in Tornadoes Experiment: VORTEX. Bull. Amer. Meteor. Soc., 75, 995-1006, doi:10.1175/1520-0477(1994)075<0995: VOTOOR $>2.0 . \mathrm{CO} ; 2$.

,-- , M. S. Gilmore, and R. Davies-Jones, 2006: A preliminary survey of rear-flank descending reflectivity cores in supercell storms. Wea. Forecasting, 21, 923-938, doi:10.1175/ WAF962.1.

Romine, G. S., D. W. Burgess, and R. B. Wilhelmson, 2008: A dualpolarization-radar-based assessment of the 8 May 2003 Oklahoma City area tornadic supercell. Mon. Wea. Rev., 136, 2849-2870, doi:10.1175/2008MWR2330.1.

Rotunno, R., and J. B. Klemp, 1982: The influence of shearinduced pressure gradient on thunderstorm motion. Mon. Wea. Rev., 110, 136-151, doi:10.1175/1520-0493(1982)110<0136: TIOTSI $>2.0 . \mathrm{CO} ; 2$

Schenkman, A. D., M. Xue, and M. Hu, 2014: Tornadogenesis in a high-resolution simulation of the 8 May 2003 Oklahoma City supercell. J. Atmos. Sci., 71, 130-154, doi:10.1175/JAS-D-13-073.1.
Skinner, P. S., C. C. Weiss, J. L. Schroeder, L. J. Wicker, and M. I. Biggerstaff, 2011: Observations of the surface boundary structure within the 23 May 2007 Perryton, Texas, supercell. Mon. Wea. Rev., 139, 3730-3749, doi:10.1175/MWR-D-10-05078.1.

Snook, N., and M. Xue, 2008: Effects of microphysical drop size distribution on tornadogenesis in supercell thunderstorms. Geophys. Res. Lett., 35, L24803, doi:10.1029/2008GL035866.

Straka, J. M., E. N. Rasmussen, and S. E. Fredrickson, 1996: A mobile mesonet for finescale meteorological observations. J. Atmos. Oceanic Technol., 13, 921-936, doi:10.1175/ 1520-0426(1996)013<0921:AMMFFM > 2.0.CO;2.

,,-- R. P. Davies-Jones, and P. M. Markowski, 2007: An observational and idealized numerical examination of lowlevel counter-rotating vortices toward the rear flank of supercells. Electron. Severe Storms Meteor., 2 (8). [Available online at http://www.ejssm.org/ojs/index.php/ejssm/article/ viewArticle/32.]

Tanamachi, R. L., H. B. Bluestein, M. Xue, W.-C. Lee, K. A. Orzel, S. J. Frasier, and R. M. Wakimoto, 2013: Near-surface vortex structure in a tornado and in a sub-tornado-strength convective-storm vortex observed by a mobile, W-band radar during VORTEX2. Mon. Wea. Rev., 141, 3661-3690, doi:10.1175/MWR-D-12-00331.1.

Trapp, R. J., and C. A. Doswell, 2000: Radar data objective analysis. J. Atmos. Oceanic Technol., 17, 105-120, doi:10.1175/ 1520-0426(2000)017<0105:RDOA > 2.0.CO;2.

Wakimoto, R. M., and H. Cai, 2000: Analysis of a nontornadic storm during VORTEX 95. Mon. Wea. Rev., 128, 565-592, doi:10.1175/1520-0493(2000)128<0565:AOANSD>2.0.CO;2. C. Liu, and H. Cai, 1998: The Garden City, Kansas, storm during VORTEX 95. Part I: Overview of the storm life cycle and mesocyclogenesis. Mon. Wea. Rev., 126, 372-392, doi:10.1175/1520-0493(1998)126<0372:TGCKSD>2.0.CO;2.

- H. V. Murphey, D. C. Dowell, and H. B. Bluestein, 2003: The Kellerville tornado during VORTEX: Damage survey and Doppler radar analysis. Mon. Wea. Rev., 131, 2197-2221, doi:10.1175/1520-0493(2003)131<2197:TKTDVD>2.0.CO;2.

, N. T. Atkins, and J. Wurman, 2011: The LaGrange tornado during VORTEX2. Part I: Photogrammetric analysis of the tornado combined with single-Doppler radar data. Mon. Wea. Rev., 139, 2233-2258, doi:10.1175/2010MWR3568.1.

Waugh, S., and S. E. Fredrickson, 2010: An improved aspirated temperature system for mobile meteorological observations, especially in severe weather. 25th Conf. on Severe Local Storms, Denver, CO, Amer. Meteor. Soc., P5.2. [Available online at https://ams.confex.com/ams/25SLS/webprogram/ Paper176205.html.]

Weiss, C. C., and J. L. Schroeder, 2008: StickNet: A new portable, rapidly-deployable surface observation system. Bull. Amer. Meteor. Soc., 89, 1502-1503.

J. Guynes, P. S. Skinner, and J. Beck, 2009: The TTUKa mobile Doppler radar: Coordinated radar and in situ measurements of supercell thunderstorms during Project VORTEX2. 34th Conf. on Radar Meteorology, Williamsport, VA, Amer. Meteor. Soc., 11B.2. [Available online at https://ams.confex. com/ams/34Radar/techprogram/paper_155425.htm.]

, D. C. Dowell, P. S. Skinner, and A. E. Reinhart, 2012: An assessment of buoyancy and baroclinity within select VORTEX2 storms. 26th Conf. on Severe Local Storms, Nashville, TN, Amer. Meteor. Soc., 13.4. [Available online at https://ams. confex.com/ams/26SLS/webprogram/Paper211795.html.]

Wicker, L. J., and R. B. Wilhelmson, 1995: Simulation and analysis of tornado development and decay within a three-dimensional 
supercell thunderstorm. J. Atmos. Sci., 52, 2675-2703, doi:10.1175/1520-0469(1995)052<2675:SAAOTD>2.0.CO;2.

Wurman, J., and S. Gill, 2000: Finescale radar observations of the Dimmit, Texas (2 June 1995), tornado. Mon. Wea. Rev., 128, 2135-2164, doi:10.1175/1520-0493(2000)128<2135: FROOTD $>2.0 . \mathrm{CO} ; 2$.

_, and K. Kosiba, 2013: Fine-scale radar observations of tornado and mesocyclone structures. Wea. Forecasting, 28, 1157-1175, doi:10.1175/WAF-D-12-00127.1.

, J. M. Straka, E. N. Rasmussen, M. Randall, and A. Zahrai, 1997: Design and deployment of a portable, pencil-beam, pulsed, 3-cm Doppler radar. J. Atmos. Oceanic Technol., 14, 1502-1512, doi:10.1175/1520-0426(1997)014<1502:DADOAP>2.0.CO;2.

, Y. P. Richardson, C. Alexander, S. Weygant, and P. F. Zhang, 2007: Dual-Doppler and single-Doppler analysis of a tornadic storm undergoing mergers and repeated tornadogenesis. Mon. Wea. Rev., 135, 736-758, doi:10.1175/MWR3276.1.
—_, K. Kosiba, P. Markowski, Y. P. Richardson, D. Dowell, and P. Robinson, 2010: Finescale single- and dualDoppler analysis of tornado intensification, maintenance, and dissipation in the Orleans, Nebraska, supercell. Mon. Wea. Rev., 138, 4439-4455, doi:10.1175/ 2010MWR3330.1.

—, D. Dowell, Y. P. Richardson, P. Markowski, E. Rasmussen, D. Burgess, L. Wicker, and H. Bluestein, 2012: The second Verification of the Origins of Rotation in Tornadoes Experiment: VORTEX2. Bull. Amer. Meteor. Soc., 93, 1147-1170, doi:10.1175/BAMS-D-11-00010.1.

Ziegler, C. L., E. N. Rasmussen, T. R. Shepherd, A. I. Watson, and J. M. Straka, 2001: The evolution of low-level rotation in the 29 May 1994 Newcastle-Graham, Texas, storm complex during VORTEX. Mon. Wea. Rev., 129, 1339-1368, doi:10.1175/1520-0493(2001)129<1339: TEOLLR $>2.0 . \mathrm{CO} ; 2$. 